\title{
Leptogenesis after superconformal subcritical hybrid inflation
}

\section{Yoshihiro Gunji and Koji Ishiwata}

Institute for Theoretical Physics, Kanazawa University, Kanazawa 920-1192, Japan

E-mail: y_gunji@hep.s.kanazawa-u.ac.jp, ishiwata@hep.s.kanazawa-u.ac.jp

ABSTRACT: We consider an extended version of superconformal subcritical hybrid inflation model by introducing three right-handed neutrinos that have the Majorana mass terms. In the model one of the right-handed sneutrinos plays a role of the inflaton field, and it decays to reheat the universe after inflation. The vacuum expectation value for the waterfall field gives an unconventional pattern of the light neutrino mass matrix, and the neutrino Yukawa couplings that determine the reheating temperature are not constrained by the neutrino oscillation data. Consequently thermal leptogenesis or sneutrino leptogenesis is realized.

Keywords: Cosmology of Theories beyond the SM, CP violation, Neutrino Physics, Supergravity Models

ARXiv EPRINT: 1906.04530 


\section{Contents}

1 Introduction 1

2 The model 2

3 Inflation 4

3.1 Pre-critical regime 5

$\begin{array}{lll}3.2 & \text { Subcritical regime } & 6\end{array}$

$\begin{array}{lll}3.3 & \text { Stability of inflationary trajectory } & 7\end{array}$

4 Neutrino mass $\quad 8$

4.1 Mass matrix 8

$\begin{array}{ll}4.2 \text { Parametrization of neutrino Yukawa couplings } & 10\end{array}$

5 Post inflationary regime $\quad 12$

$\begin{array}{lll}5.1 \text { Reheating } & 12\end{array}$

$\begin{array}{lll}5.2 & \text { Leptogenesis } & 13\end{array}$

$\begin{array}{lll}5.3 & \text { Gravitino problem } & 18\end{array}$

6 Conclusion 21

\section{Introduction}

Inflation paradigm is strongly supported by the observations of the cosmic microwave background radiation (CMB). Slow-roll scalar field in the early universe is a promising candidate for inflation, and many types of inflation models have been proposed so far. In a theoretical point of view, it would be tempting to ask what the underlying physics or symmetry of the inflaton field is. Supersymmetry (SUSY) might be one of the answers. It protects the flatness of the inflaton direction, which is suitable for inflation.

Recently supersymmetric D-term hybrid inflation has been revisited in various point of view. Under shift symmetric Kähler potential [1], subcritical hybrid inflation was found, where inflation continues for subcritical point value of the inflaton field $[2,3]$. On the other hand, it was shown in refs. $[4,5]$ that Starobinsky model [6] emerges in the framework of superconformal supergravity [7-10]. It turned out in the following study that this framework has another new regime of inflation. It was shown that a general class of superconformal $\alpha$-attractor model $[11,12]$ appears in the subcritical regime of inflation, which we call superconformal subcritical hybrid inflation [13]. In addition, the energy scale of inflation should coincide with the grand unification scale to be consistent with the Planck observation data, which is the feature found in the subcritical hybrid inflation $[2,3]$. 
Namely, the superconformal subcritical hybrid inflation has both features of the superconformal $\alpha$-attractor models and the subcritical hybrid inflation. The shift symmetry and superconformality are crucial for them.

In this paper we will study the thermal history after the end of superconformal subcritical hybrid inflation. (See refs. $[14,15]$ that study the phenomenology of Pati-Salam version of subcritical hybrid inflation. Recently ref. [16] comprehensively studies the D-term hybrid inflation, including reheating, leptogenesis, and the SUSY breaking mechanism.) For the purpose, we introduce three right-handed neutrinos that interact with the minimal supersymmetric standard model (MSSM) sector. In fermionic sector, the mass matrix for the light neutrinos is given by the seesaw mechanism [17-22], but it has an unconventional structure. In bosonic sector, on the other hand, it will be shown that one of sneutrinos can play a role of the inflaton field. In addition, baryon asymmetry that is sufficient amount to explain the observed value is generated via leptogenesis [23].

This paper is organized as follows. In the next section the model that we consider is described. Then section 3 shows the conditions required for the superconformal subcritical hybrid inflation in this model. Mass matrices of the heavy and light (s)neutrinos, including parametrization of the neutrino Yukawa couplings, are given in section 4, then we discuss the reheating and leptogenesis after inflation in section 5. Section 6 is dedicated to conclusion.

\section{The model}

We consider a model described by the superpotential

$$
W=W_{\mathrm{MSSM}}+W_{\text {neu }},
$$

where $W_{\text {MSSM }}$ is the superpotential of the MSSM sector and

$$
W_{\text {neu }}=\frac{1}{2} M_{i j} N_{i}^{c} N_{j}^{c}+y_{\nu i j} N_{i}^{c} L_{j} H_{u}+\lambda_{i} N_{i}^{c} S_{+} S_{-} .
$$

Here $N_{i}^{c}, L_{i}=\left(\nu_{L i}, l_{L i}\right)^{T}$, and $H_{u}=\left(H_{u}^{+}, H_{u}^{0}\right)^{T}$ are the chiral superfields of the righthanded neutrinos, left-handed leptons, and up-type Higgs, respectively, and $L_{j} H_{u}=$ $\nu_{L j} H_{u}^{0}-l_{L j} H_{u}^{+} . S_{ \pm}$are the local U(1) fields with charge $\pm q(q>0)$, one of which plays a role of the waterfall field. ${ }^{1}$ Indices are summed over $i, j=1-3$. (We will use the same contraction in the following discussion unless otherwise mentioned.) $M_{i j}$ terms are explicit superconformal breaking terms that are added by phenomenological purpose. While we do not argue their origin, $M_{i j} \ll 1$ are expected. The Kähler potential, on the other hand, is given by

$$
\mathcal{K}=-3 \log \Omega^{-2},
$$

\footnotetext{
${ }^{1}$ We write the superpartners with tilde for the MSSM fields and right-handed neutrinos. For $S_{ \pm}$, the same symbols are used for scalar fields while fermionic parts are expressed with tilde. In the current and the next sections, we adopt the unit in which the reduced Planck mass $M_{p l} \simeq 2.4 \times 10^{18} \mathrm{GeV}$ is taken to be unity unless otherwise mentioned.
} 
where

$$
\Omega^{-2}=1-\frac{1}{3}\left(\sum_{\mathrm{MSSM}}\left|I_{\mathrm{MSSM}}\right|^{2}+\sum_{i}\left|N_{i}^{c}\right|^{2}+\left|S_{+}\right|^{2}+\left|S_{-}\right|^{2}\right)-\sum_{i} \frac{\chi_{i}}{6}\left(N_{i}^{c 2}+\bar{N}_{i}^{c 2}\right) .
$$

Here $I_{\text {MSSM }}$ are chiral superfields in the MSSM sector. The last term is superconformal breaking term that is considered in refs. [4, 10]. With the superpotential and Kähler potential, the scalar potential is given by

$$
V_{\mathrm{tot}}=V_{F}+V_{D}
$$

where $V_{F}$ and $V_{D}$ are $\mathrm{F}$ - and D-terms, respectively, and given by [4]

$$
\begin{aligned}
& V_{F}=\Omega^{4}\left[\delta^{\alpha \bar{\alpha}} W_{\alpha} W_{\bar{\alpha}}+\frac{1}{\Delta}\left|\delta^{\alpha \bar{\alpha}} W_{\alpha} \Phi_{\bar{\alpha}}-3 W\right|^{2}\right], \\
& V_{D}=\frac{1}{2} g^{2}\left[q \Omega^{2}\left(\left|S_{+}\right|^{2}-\left|S_{-}\right|^{2}\right)-\xi\right]^{2} .
\end{aligned}
$$

Here $\Phi \equiv-3 \Omega^{-2}$ and $\Delta \equiv \Phi-\delta^{\alpha \bar{\alpha}} \Phi_{\alpha} \Phi_{\bar{\alpha}}$ have been additionally introduced. Subscript in $W$ and $\Phi$ stands for the field derivative, e.g., $W_{\alpha} \equiv \partial W / \partial z^{\alpha}$ where $z^{\alpha}$ is a chiral superfield. In the D-term, we have introduced the Fayet-Iliopoulos (FI) term $\xi(>0)$ associated with the U(1). ${ }^{2}$ Due to the FI term, $S_{+}$has a vacuum expectation value (VEV) at the global minimum, which is obtained as $\left\langle S_{+}\right\rangle=\sqrt{\xi / q(1+\tilde{\xi})}$ with $\tilde{\xi} \equiv \xi / 3 q$.

As in ref. [13], we take $\chi_{i} \leq 0$ without loss of generality. In the present model, we impose the following condition:

$$
\chi_{3} \simeq-1, \quad \chi_{1}, \chi_{2} \simeq 0 .
$$

This distinguishes $N_{3}^{c}$ from $N_{1}^{c}$ and $N_{2}^{c} . \phi \equiv \sqrt{2} \operatorname{Re} \tilde{N}_{3}^{c}$ has an approximate shift symmetry that is explicitly broken by $\lambda_{3}(\ll 1)$. Then, $\phi$ is expected to be the inflaton as studied in ref. [13]. In the inflation model, $s \equiv \sqrt{2}\left|S_{+}\right|$plays a role of the waterfall field. $N_{1}^{c}$ and $N_{2}^{c}$, on the other hand, have no such symmetry. Instead there is a freedom to choose any basis for $N_{1}^{c}$ and $N_{2}^{c}$ with a redefinition of $M_{i j}$ and $\lambda_{i}$ due to $\chi_{1,2} \simeq 0$.

For simple notation, hereafter we omit subscripts of $\chi_{3}$ and $\lambda_{3}$ and introduce $m_{\phi}$, which will be identified as the inflaton mass in section 3.2 (with another assumption in section 4.1),

$$
\begin{aligned}
\chi & \equiv \chi_{3}, \quad \lambda \equiv \lambda_{3}, \\
m_{\phi} & \equiv \lambda\left\langle S_{+}\right\rangle .
\end{aligned}
$$

It is sometimes convenient to use $\delta \chi(0<\delta \chi<1)$ defined by $\delta \chi /(1+\chi)=-q g^{2} \xi / 3 \lambda^{2}$. $0<\delta \chi<1$ guarantees $\Omega(\phi, s)^{2}>0$ and $\phi_{c, 0}^{2}>0$, which will be defined in eqs. (3.2)

\footnotetext{
${ }^{2}$ The origin of the FI term in canonical superconformal supergravity model [10] is discussed in ref. [4]. See also ref. [16] for recent development.
} 
and (3.4), respectively. Then inflation that is consistent with the observations of the CMB is realized in the parameter space [13],

$$
\begin{aligned}
\lambda & \simeq(0.5-1) \times 10^{-3}, \quad \xi^{1 / 2} \simeq(3-1) \times 10^{16} \mathrm{GeV}, \\
m_{\phi} & \simeq(1-2) \times 10^{13} \mathrm{GeV},
\end{aligned}
$$

for $q=g=1, \delta \chi=0.9$ and the number of $e$-folds $N_{e}=55-60$, which we take in the later numerical study. ${ }^{3}$ In the parameter space, e.g., $\chi \simeq-1.16$ for $\lambda=10^{-3}, \xi^{1 / 2}=10^{16} \mathrm{GeV}$, and $\delta \chi=0.9$. The mass terms in the superpotential, however, have a possibility to alter the inflationary path. In the next section, we will derive the conditions in order not to affect the inflationary dynamics.

\section{Inflation}

We define several variables that are used in the following analysis. During inflation, the other fields except for the inflaton and waterfall field are irrelevant. Thus it is convenient to define following potentials,

$$
\begin{aligned}
& \left.V(\phi, s) \equiv V_{\text {tot }}\right|_{\sqrt{2} \operatorname{Re} \tilde{N}_{3}^{c}=\phi, \sqrt{2}\left|S_{+}\right|=s, \text { the others=0 }}, \\
& \left.\Omega(\phi, s) \equiv \Omega\right|_{\sqrt{2} \operatorname{Re} \tilde{N}_{3}^{c}=\phi, \sqrt{2}\left|S_{+}\right|=s, \text { the others=0 }}
\end{aligned}
$$

Then the critical point value $\phi_{c}$ is defined as a field value below which the waterfall field becomes tachyonic. It receives $\mathcal{O}\left(M_{i j}^{2}\right)$ corrections as

$$
\phi_{c}^{2}=\phi_{c, 0}^{2}\left[1-\frac{2 \Delta M^{2}\left(\phi_{c, 0}\right)}{3 \lambda^{2}}\right]+\mathcal{O}\left(M_{i 3}^{4}\right)
$$

where

$$
\begin{aligned}
\phi_{c, 0}^{2} & =\frac{6 q g^{2} \xi}{3 \lambda^{2}+(1+\chi) q g^{2} \xi} \\
\Delta M^{2}(\phi) & =\sum_{i=1}^{3}\left|M_{i 3}\right|^{2}-\frac{(1-2 \chi)^{2}}{24} \frac{\phi^{2}}{1+\phi^{2} \chi(1+\chi) / 6}\left|M_{33}\right|^{2} .
\end{aligned}
$$

For example, $\phi_{c, 0} \simeq 18$ or in terms of canonically-normalized field $\hat{\phi}_{c, 0} \simeq 11$ defined in eq. (3.12) for $\lambda=10^{-3}, \xi^{1 / 2}=10^{16} \mathrm{GeV}$ and $\delta \chi=0.9$. This perturbative expansion is valid when ${ }^{4}$

$$
\begin{aligned}
\left|M_{13}\right|^{2}+\left|M_{23}\right|^{2} & \ll 3 \lambda^{2} / 2 \sim\left(10^{15} \mathrm{GeV}\right)^{2}, \\
\left|M_{33}\right|^{2} & \ll 2 \lambda^{4} / q g^{2} \xi \sim\left(10^{14} \mathrm{GeV}\right)^{2} .
\end{aligned}
$$

It will be checked in this section that the above conditions are satisfied in this inflation model.

\footnotetext{
${ }^{3}$ We will estimate the number of $e$-folds in section 5.1 to confirm this.

${ }^{4}$ We have checked that $\mathcal{O}\left(M_{i 3}^{4}\right)$ term is irrelevant when eq. (3.7) is satisfied, thus we ignore it in the following discussion.
} 
Finally it is useful to define

$$
\Psi \equiv \frac{\Omega(\phi, 0) \phi}{\Omega\left(\phi_{c, 0}, 0\right) \phi_{c, 0}}=\frac{\Omega(\phi, 0) \phi}{\sqrt{2 q g^{2} \xi / \lambda^{2}}}
$$

when potential is expressed in terms of canonically-normalized inflaton field.

\subsection{Pre-critical regime}

Let us begin with the regime where the inflaton is approaching down to the critical point value. Since the waterfall field is stabilized at the origin in this regime, the relevant Lagrangian is given as

$$
\mathcal{L}_{\text {pre }}=\frac{f(\phi, 0)}{2}\left(\partial_{\mu} \phi\right)^{2}-V_{\text {pre }}(\phi)
$$

where $^{5}$

$$
\begin{aligned}
f(\phi, s) & =\Omega^{2}(\phi, s)\left[1+\Omega^{2}(\phi, s) \frac{(1+\chi)^{2}}{6} \phi^{2}\right], \\
V_{\text {pre }}(\phi) & =\frac{1}{2} g^{2} \xi^{2}\left[1+2 \Psi^{2} \frac{\Omega^{2}(\phi, 0) \Delta M^{2}(\phi)}{\lambda^{2} \xi / q}\right] .
\end{aligned}
$$

It is seen that $\Delta M^{2}$ term gives a gradient to the inflaton field, which should not invade the slow-roll conditions. To see the impact of $\Delta M^{2}$ term, it is instructive to change dynamical variable $\phi$ to canonically-normalized field $\hat{\phi}$. Since $\chi \simeq-1$, it is a good approximation that $f(\phi, 0) \simeq \Omega^{2}(\phi, 0)$. Then $d \phi / d \hat{\phi}=f(\phi)^{-1 / 2} \simeq \Omega^{-1}(\phi, 0)$ can be solved easily to obtain,

$$
\begin{aligned}
\phi & \simeq \beta^{-1 / 2} \sinh \beta^{1 / 2} \hat{\phi}, \\
\Psi & \simeq \delta \chi^{-1 / 2} \tanh \beta^{1 / 2} \hat{\phi}, \\
\Omega^{-1}(\phi, 0) & \simeq \cosh \beta^{1 / 2} \hat{\phi},
\end{aligned}
$$

where $\beta=-(1+\chi) / 6=\lambda^{2} \delta \chi / 2 q g^{2} \xi$. Then the potential in terms of $\hat{\phi}$ is given as

$$
V_{\text {pre }} \simeq \frac{1}{2} g^{2} \xi^{2}\left[1+\frac{\tanh ^{2} \beta^{1 / 2} \hat{\phi}}{\cosh ^{2} \beta^{1 / 2} \hat{\phi}} \frac{2 q}{\lambda^{2} \xi \delta \chi}\left\{\sum_{i=1}^{3}\left|M_{i 3}\right|^{2}-\frac{3}{8 \beta}\left|M_{33}\right|^{2} \tanh ^{2} \beta^{1 / 2} \hat{\phi}\right\}\right] .
$$

On the other hand, it was shown in ref. [4] that there is one-loop corrections to the tree-level potential. In terms of the canonically-normalized field, it is given by

$$
V_{1 l} \simeq \frac{1}{2} g^{2} \xi^{2} \times \frac{q^{2} g^{2}}{8 \pi^{2}} \log \left[\delta \chi^{-1} \tanh ^{2} \beta^{1 / 2} \hat{\phi}\right] .
$$

Therefore, in order not to affect the inflationary trajectory, it is sufficient that the terms proportional to $\left|M_{i 3}\right|^{2}$ are subdominant compared to the one-loop potential. In the parameter space given in eq. $(2.11), \sqrt{\beta} \hat{\phi}_{c} \simeq \operatorname{arcsinh} \sqrt{\beta} \phi_{c} \sim \mathcal{O}(1)$. Then, the conditions are

\footnotetext{
${ }^{5}$ It is noted that the term proportional to $\Delta M^{2}$ in eq. (3.11) is equivalent to $\Omega^{4}(\phi, 0) \phi^{2} \Delta M^{2}(\phi) / 2$.
} 
given as

$$
\begin{gathered}
\left|M_{13}\right|^{2}+\left|M_{23}\right|^{2} \lesssim \frac{q g^{2} \delta \chi \lambda^{2} \xi}{16 \pi^{2}} \lesssim\left(1 \times 10^{12} \mathrm{GeV}\right)^{2} \\
\left|M_{33}\right|^{2} \lesssim \frac{\delta \chi^{2} \lambda^{4}}{12 \pi^{2}} \lesssim\left(2 \times 10^{11} \mathrm{GeV}\right)^{2}
\end{gathered}
$$

It is easy to check that the slow-roll conditions are satisfied under the constraints. Since the constraints are more stringent than eqs. (3.6) and (3.7), it has been confirmed that the perturbative expansion to obtain eq. (3.3) is valid.

\subsection{Subcritical regime}

In the previous subsection, we have seen that the slow-roll conditions are satisfied before reaching to the critical point value. After the inflaton field becomes subcritical point value, the tachyonic growth of the waterfall field occurs. It is expected that the inflation continues in the subcritical regime when $M_{i 3} \rightarrow 0$. In this subsection, we will derive the conditions under which the inflaton and waterfall field dynamics are not affected with non-zero $M_{i 3}$.

As seen in the previous section, the perturbative expression for $\phi_{c}$ is valid under the conditions given in eqs. (3.17) and (3.18). Then, the tachyonic growth of the waterfall field is not affected by the additional gradient in the inflaton direction due to $\left|M_{i 3}\right|^{2}$ terms since $\phi_{c} \simeq \phi_{c, 0}$. Consequently, the dynamics of the waterfall field around the critical point is the same as one discussed in ref. [13]. The tachyonic growth is qualitatively the same as the subcritical hybrid inflation [2,3]. Namely, due to the tachyonic growth, the waterfall field relaxes to the local minimum value $s_{\min }$ just after a few Hubble-unit time. (See, for example, figure 1 of ref. [2].) In the present model, $s_{\min }$ is found to be

$$
s_{\min }^{2}=\frac{2 \xi}{q(1+\tilde{\xi})} \frac{\Omega^{-2}(\phi, 0)}{1+\tilde{\xi} \Psi^{2} /(1+\tilde{\xi})}\left[1-\Psi^{2}\left\{1+\frac{2 \Omega^{2}(\phi, 0) \Delta M^{2}(\phi)}{3 \lambda^{2}}\right\}\right] .
$$

Then the potential in the subcritical regime of the inflaton field is effectively given by $V\left(\phi, s_{\min }\right)$ and the dynamics reduces to single field inflation that is described by the Lagrangian,

$$
\mathcal{L}=\frac{f\left(\phi, s_{\min }\right)}{2}\left(\partial_{\mu} \phi\right)^{2}-V_{\text {inf }}(\phi)
$$

where

$$
\begin{aligned}
V_{\mathrm{inf}}(\phi)= & g^{2} \xi^{2} \frac{(1+\tilde{\xi}) \Psi^{2}}{1+2 \tilde{\xi} \Psi^{2}}\left[1-\frac{\Psi^{2}}{2(1+\tilde{\xi})}+\frac{\left\{1+\tilde{\xi} \Psi^{2} /(1+\tilde{\xi})\right\}^{2}}{\left(1+2 \tilde{\xi} \Psi^{2}\right) /(1+\tilde{\xi})} \frac{\Omega^{2}(\phi, 0) \Delta M^{2}(\phi)}{\lambda^{2} \xi / q}\right] \\
& +\mathcal{O}\left(M_{i 3}^{4}\right) .
\end{aligned}
$$

Recall that $\tilde{\xi}, \xi \ll 1$ in the parameters in eq. (2.11). Then it is clear that the additional term proportional to $\Delta M^{2}$ is the same as in eq. (3.11). Note that $s_{\min }$ is negligible in $\Omega\left(\phi, s_{\min }\right)$. Then, the canonically-normalized inflaton field is given by eqs. (3.12)-(3.14). 
Consequently, $V_{\text {inf }}$ is given by

$$
\begin{aligned}
V_{\mathrm{inf}} \simeq & g^{2} \xi^{2} \delta \chi^{-1} \tanh ^{2} \beta^{1 / 2} \hat{\phi}\left[1-\frac{\delta \chi^{-1}}{2} \tanh ^{2} \beta^{1 / 2} \hat{\phi}\right. \\
& \left.+\frac{\cosh ^{-2} \beta^{1 / 2} \hat{\phi}}{\lambda^{2} \xi / q}\left\{\sum_{i=1}^{3}\left|M_{i 3}\right|^{2}-\frac{3}{8 \beta}\left|M_{33}\right|^{2} \tanh ^{2} \beta^{1 / 2} \hat{\phi}\right\}\right] .
\end{aligned}
$$

Therefore, if eqs. (3.17) and (3.18) are satisfied, then the dynamics in the subcritical regime reduces to one in ref. [13]. In addition, it is clear that $m_{\phi}$ defined in eq. (2.10) is indeed the inflaton mass since $V_{\text {inf }} \simeq \frac{1}{2} m_{\phi}^{2} \phi^{2}$ around $\phi(\simeq \hat{\phi}) \sim 0$.

To summarize the present and previous subsections, the inflaton-waterfall field dynamics is unchanged when

$$
\left|M_{i 3}\right|^{2}=\left|M_{3 i}\right|^{2} \lesssim\left(2 \times 10^{11} \mathrm{GeV}\right)^{2}
$$

for $i=1-3$ are satisfied.

\subsection{Stability of inflationary trajectory}

It was pointed out in ref. [24] that $\tilde{L}_{i} H_{u}$ may become tachyonic in sneutrino inflation. In order to find out the stability condition, let us derive the mass matrix in $\tilde{L}_{i}$ and $H_{u}$ basis. From $V_{\text {tot }}$, it is obtained by

$$
\begin{aligned}
\mathcal{L}_{\text {mass }}^{\tilde{L}-H_{u}}= & -\frac{\Omega(\phi, s)^{4} \phi^{2}}{2}\left|y_{\nu 3 i} \tilde{L}_{i}\right|^{2}-\frac{\Omega(\phi, s)^{4} \phi^{2}}{2}\left|y_{\nu 3 i}\right|^{2}\left|H_{u}\right|^{2} \\
& +\left[\frac{M_{33}^{*} \Omega(\phi, s)^{6} \phi^{3}}{4 \sqrt{2}} y_{\nu 3 i} \tilde{L}_{i} H_{u}+\text { h.c. }\right] .
\end{aligned}
$$

On the other hand, the kinetic terms of $\tilde{L}_{i}$ and $H_{u}$ are given by

$$
\mathcal{L}_{\text {kin }}^{\tilde{L}-H_{u}}=\Omega(\phi, s)^{2}\left[\left|\partial_{\mu} \tilde{L}_{i}\right|^{2}+\left|\partial_{\mu} H_{u}\right|^{2}\right] .
$$

Therefore, using canonically-normalized fields, $\hat{\tilde{L}}_{i} \equiv \Omega(\phi, s) \tilde{L}_{i}$ and $\hat{H}_{u} \equiv \Omega(\phi, s) H_{u}$, the mass terms are rewritten as

$$
\begin{aligned}
\mathcal{L}_{\text {mass }}^{\tilde{L}-H_{u}}= & -\frac{\Omega(\phi, s)^{2} \phi^{2}}{2}\left|y_{\nu 3 i} \hat{\tilde{L}}_{i}\right|^{2}-\frac{\Omega(\phi, s)^{2} \phi^{2}}{2}\left|y_{\nu 3 i}\right|^{2}\left|\hat{H}_{u}\right|^{2} \\
& +\left[\frac{M_{33}^{*} \Omega(\phi, s)^{4} \phi^{3}}{4 \sqrt{2}} y_{\nu 3 i} \hat{\tilde{L}}_{i} \hat{H}_{u}+\text { h.c. }\right] \\
= & -\frac{\Omega(\phi, s)^{2} \phi^{2}}{2}\left(y_{\nu} y_{\nu}^{\dagger}\right)_{33}\left|\hat{\tilde{L}}_{3}^{\prime}\right|^{2}-\frac{\Omega(\phi, s)^{2} \phi^{2}}{2}\left(y_{\nu} y_{\nu}^{\dagger}\right)_{33}\left|\hat{H}_{u}\right|^{2} \\
& +\left[\frac{M_{33}^{*} \Omega(\phi, s)^{4} \phi^{3}}{4 \sqrt{2}}\left(y_{\nu} y_{\nu}^{\dagger}\right)_{33}^{1 / 2} \hat{\tilde{L}}_{3}^{\prime} \hat{H}_{u}+\text { h.c. }\right]
\end{aligned}
$$

where we have defined $\left(y_{\nu} y_{\nu}^{\dagger}\right)_{33}^{1 / 2} \hat{\tilde{L}}_{3}^{\prime} \equiv y_{\nu 3 i} \hat{\tilde{L}}_{i}$ in the second line following ref. [24]. Then, the stability condition is given by

$$
\left|M_{33}\right|<2 \sqrt{2}\left(y_{\nu} y_{\nu}^{\dagger}\right)_{33}^{1 / 2} \frac{\Omega(\phi, s)^{-2}}{\phi} .
$$


Using eqs. (3.12) and (3.14), it turns out that

$$
\begin{aligned}
\left|M_{33}\right| & <4 \sqrt{2}\left(y_{\nu} y_{\nu}^{\dagger}\right)_{33}^{1 / 2} \sqrt{\beta} \\
& \simeq 1.4 \times 10^{14} \mathrm{GeV}\left(\frac{\left(y_{\nu} y_{\nu}^{\dagger}\right)_{33}}{10^{-7}} \frac{\beta}{10^{-3}}\right)^{1 / 2} .
\end{aligned}
$$

This upper bound is weaker than (3.23) in most of the parameter space, which will be seen later.

\section{Neutrino mass}

In this section, we derive the mass matrices for the heavy and light neutrinos. Around the global minimum, $\Omega \simeq 1$ since $\xi \ll 1$. Consequently, all the fields are canonical. Thus, the mass terms are derived similarly in global SUSY model.

\subsection{Mass matrix}

The superpotential (2.2) gives the Majorana masses for the light neutrinos. To see how the masses are generated, we write down the mass terms for fermionic part of $N_{i}^{c}, \nu_{L i}$ and $\tilde{S}_{-}$,

$$
\mathcal{L}_{\nu}^{\text {mass }}=-\frac{1}{2}\left(\bar{\psi} \mathcal{M} P_{L} \psi+\text { h.c. }\right),
$$

where

$$
\begin{aligned}
\psi & =\left(N_{1}^{c}, N_{2}^{c}, N_{3}^{c}, \tilde{S}_{-}, \nu_{L 1}, \nu_{L 2}, \nu_{L 3}\right)^{T}, \\
\mathcal{M} & =\left(\begin{array}{cc}
\tilde{M} & \tilde{m}_{\nu} \\
\tilde{m}_{\nu}^{T} & \mathbf{0}
\end{array}\right) .
\end{aligned}
$$

$\tilde{M}$ and $\tilde{m}_{\nu}$ are $4 \times 4$ and $4 \times 3$ matrices, respectively, and given by

$$
\begin{aligned}
& \tilde{M}=\left(\begin{array}{cc} 
& \lambda_{1}\left\langle S_{+}\right\rangle \\
M & \lambda_{2}\left\langle S_{+}\right\rangle \\
& m_{\phi} \\
\lambda_{1}\left\langle S_{+}\right\rangle \lambda_{2}\left\langle S_{+}\right\rangle m_{\phi} & 0
\end{array}\right), \\
& \tilde{m}_{\nu}=\left(\begin{array}{ccc} 
& & \\
& m_{\nu} & \\
& & \\
0 & 0 & 0
\end{array}\right) \text {. }
\end{aligned}
$$

Here $m_{\nu i j}=y_{\nu i j}\left\langle H_{u}^{0}\right\rangle$ with $\left\langle H_{u}^{0}\right\rangle$ being the VEV of the up-type neutral Higgs. Then mass matrix $M_{\nu}$ for the light neutrinos are obtained by the seesaw mechanism [17-22],

$$
M_{\nu}=-\tilde{m}_{\nu}^{T} \tilde{M}^{-1} \tilde{m}_{\nu}
$$

An important consequence of the mass matrix is that the one of three light neutrinos is massless. This is because the rank of $M_{\nu}$ is two. Using this mass matrix, it is possible to constrain the parameters by the observed neutrino masses. 
In the later discussion we assume

$$
\lambda_{1}, \lambda_{2} \ll \lambda .
$$

Here, recall that there is a freedom to choose a basis for $N_{1}^{c}$ and $N_{2}^{c}$. Then, $M_{12}$ can be rotated away. As a result, $M_{\nu}$ is given in the following simple expression,

$$
M_{\nu i j}=-\left\langle H_{u}^{0}\right\rangle^{2} \sum_{k=1}^{2} \frac{y_{\nu k i} y_{\nu k j}}{M_{k k}}+\mathcal{O}\left(\frac{\lambda_{1,2}}{\lambda} \frac{m_{\nu i l} m_{\nu j m}}{M_{k k}}\right) .
$$

Here in the second term of right-hand side, $k=1$ or 2 and $i, j, l, m$ can be $1-3$, and we have ignored $M_{i 3}$ based on the discussion of the previous section. The leading order term, on the other hand, is independent of $\lambda_{1,2}, M_{i 3}, m_{\phi}$ and $y_{\nu 3 i}(i=1-3)$. Therefore, they are not constrained by the neutrino oscillation data. This fact is important in the estimation of the reheating temperature, which we will see later. To ensure the non-zero $\lambda_{1,2}$ does not affect our later analysis at percent level, we implicitly assume $\lambda_{1,2}<0.01 \lambda$.

Before further discussing the light neutrino mass matrix, let us note that the mass matrix $\tilde{M}$ corresponds to the mass matrix in the superpotential around the global minimum,

$$
W_{\text {neu }}=\frac{1}{2}\left(N_{1}^{c}, N_{2}^{c}, N_{3}^{c}, S_{-}\right) \tilde{M}\left(\begin{array}{c}
N_{1}^{c} \\
N_{2}^{c} \\
N_{3}^{c} \\
S_{-}
\end{array}\right)+\cdots .
$$

Recall that we have the requirement (3.23) for successful inflation. Therefore, $\tilde{M}$ should be almost block-diagonal as

$$
\tilde{M} \simeq\left(\begin{array}{ccc:c}
M_{1} & & & 0 \\
& M_{2} & & 0 \\
& & M_{3} & m_{\phi} \\
\hdashline 0 & 0 & m_{\phi} & 0
\end{array}\right),
$$

where $M_{i}>0$. Here we have left $M_{3}$ for later discussion. In the following analysis we use eq. (4.10) for $\tilde{M} . \tilde{M}$ is further diagonalized by a unitary matrix $U_{\tilde{M}}$ as,

$$
D_{\tilde{M}} \simeq \operatorname{diag}\left(M_{1}, M_{2}, m_{\phi}, m_{\phi}\right) \simeq U_{\tilde{M}}^{T} \tilde{M} U_{\tilde{M}},
$$

where

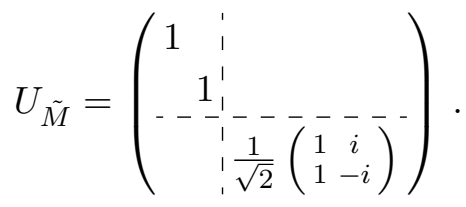

Here we have omitted $\mathcal{O}\left(M_{3} / m_{\phi}\right)$ corrections since they are irrelevant in the later analysis. Non-zero $\lambda_{1,2}$ corrections enter as $\mathcal{O}\left(\lambda_{1,2} / \lambda\right)$ and $\mathcal{O}\left(\left(m_{\phi} / M_{1,2}\right) \lambda_{1,2} / \lambda\right)$ for $m_{\phi} \gtrsim M_{1,2}$ and $m_{\phi} \lesssim M_{1,2}$, respectively. 


\begin{tabular}{|ccc|}
\hline & $\Delta m_{21}^{2}\left[10^{-5} \mathrm{eV}^{2}\right]$ & $\Delta m_{3 l}^{2}\left[10^{-3} \mathrm{eV}^{2}\right]$ \\
\hline $\mathrm{NH}$ & $7.39_{-0.20}^{+0.21}$ & $+2.525_{-0.031}^{+0.033}$ \\
$\mathrm{IH}$ & $7.39_{-0.20}^{+0.21}$ & $-2.512_{-0.031}^{+0.034}$ \\
\hline
\end{tabular}

Table 1. Neutrino mass data taken from refs. [25, 26], adopting data with the atmospheric neutrino by Super-Kamiokande. $\Delta m_{i j}^{2} \equiv m_{i}^{2}-m_{j}^{2}$, and $\Delta m_{3 l}^{2}=\Delta m_{31}^{2}>0$ for the normal hierarchy (NH) case and $\Delta m_{3 l}^{2}=\Delta m_{32}^{2}<0$ for the inverted hierarchy (IH) case.

The corrections due to non-zero $\lambda_{1,2}$ are similar in the scalar sector since the mass matrix in $\left(\tilde{N}_{1}, \tilde{N}_{2}, \tilde{N}_{3}, S_{-}\right)$basis is given by,

$$
M_{\text {scalar }}^{2}=\tilde{M}^{\dagger} \tilde{M} .
$$

Therefore the assumption (4.7) assures that the dynamics of the inflaton and the waterfall field discussed in the previous section, as well as subsequent reheating and leptogenesis, is not affected. If it is not satisfied, then the inflationary trajectory would be altered so that we need further analysis to find the paramter space that is consistent with the CMB observations. Accordingly, the subsequent reheating and leptogenesis scenario change. We leave the detailed analysis as an interesting future work.

\subsection{Parametrization of neutrino Yukawa couplings}

Now let us discuss $M_{\nu}$. It can be diagonalized by a unitary matrix $U_{\nu}$ as

$$
D_{M_{\nu}}=\operatorname{diag}\left(m_{1}, m_{2}, m_{3}\right)=U_{\nu}^{T} M_{\nu} U_{\nu} .
$$

As noted above, one of the three light neutrino masses is zero. We follow the standard convention that $m_{3}>m_{2}>m_{1}(=0)$ for the normal hierarchy $(\mathrm{NH})$ case and $m_{2}>m_{1}>$ $m_{3}(=0)$ for the inverted hierarchy $(\mathrm{IH})$ case and use the values given in refs. [25, 26], which are listed in table 1.

Before discussing the parametrization of the neutrino Yukawa couplings, it is instructive to count the number of parameters. The situation is the same as one discussed refs. $[24,27]$ since the mass matrix of the light neutrinos (4.8) is similar. Since one neutrino is massless, there are 7 parameters in low energy, i.e., 2 neutrino masses +3 real mixing angles +2 phases. On the other hand, $M_{\nu}$ includes $y_{\nu k i}$ and $M_{k}$ where $k=1,2$ and $i=1,2,3$, which means 12 real parameters (neutrino Yukawa couplings) +2 real parameters (righthanded neutrino masses). However, 3 phases can be absorbed by lepton doublets and 2 real parameters are unphysical since $M_{\nu}$ is unchanged by the rescalings $y_{\nu k i} \rightarrow \gamma_{k} y_{\nu k i}$ and $M_{k} \rightarrow \gamma_{k}^{2} M_{k}$ with $\gamma_{k}$ being real constants. Therefore, we have 9 independent parameters in $M_{\nu}$ to determine 7 parameters in the light neutrino sector. As it will be seen below, however, the parametrization of the Yukawa couplings is different, especially for $y_{\nu 3 i}$ that are important parameters for the estimation of the reheating temperature.

Let us discuss the $\mathrm{NH}$ case first. We define $4 \times 3$ matrix $R$ in the similar manner in refs. [28, 29],

$$
i R=D_{\tilde{M}}^{-1 / 2} U_{\tilde{M}}^{T} \tilde{m}_{\nu} U_{\nu} D_{M_{\nu}}^{-1 / 2}
$$


which satisfies

$$
R^{T} R=\operatorname{diag}(0,1,1)
$$

Here $D_{\tilde{M}}^{-1 / 2}$ and $D_{M_{\nu}}^{-1 / 2}$ are matrices that satisfy $\left(D_{\tilde{M}}^{-1 / 2}\right)^{2}=D_{\tilde{M}}^{-1}$ and $\left(D_{M_{\nu}}^{-1 / 2}\right)^{2}=\operatorname{diag}(0$, $\left.m_{2}^{-1}, m_{3}^{-1}\right)$, respectively. It is found that $R$ is more restrictive than eq. (4.16). Namely,

$$
\begin{aligned}
r^{T} r & =r r^{T}=\mathbf{1}, \\
R_{42} / R_{32} & =R_{43} / R_{33}=i, \\
R_{l 1} & =0 \quad(l=1-4),
\end{aligned}
$$

where

$$
r \equiv\left(\begin{array}{ll}
R_{12} & R_{13} \\
R_{22} & R_{23}
\end{array}\right) .
$$

Using the relations, the neutrino Yukawa couplings can be expressed in terms of $R$. For later discussion, it is useful to give following quantities:

$$
\begin{aligned}
\left(y_{\nu} y_{\nu}^{\dagger}\right)_{i i} & =M_{i} \sum_{j=2}^{3}\left|R_{i j}\right|^{2} m_{j} /\left\langle H_{u}^{0}\right\rangle^{2} \quad(i=1,2), \\
\left(y_{\nu} y_{\nu}^{\dagger}\right)_{33} & =2 m_{\phi} \sum_{j=2}^{3}\left|R_{3 j}\right|^{2} m_{j} /\left\langle H_{u}^{0}\right\rangle^{2} \\
\operatorname{Im}\left[\left(y_{\nu} y_{\nu}^{\dagger}\right)_{21}^{2}\right] \frac{M_{1}}{M_{2}} & =-\frac{M_{1}^{2}}{\left\langle H_{u}^{0}\right\rangle^{4}} \operatorname{Im}\left[\sum_{j=2}^{3} R_{1 j}^{2} m_{j}^{2}\right] \\
\sum_{i=1}^{2} \operatorname{Im}\left[\left(y_{\nu} y_{\nu}^{\dagger}\right)_{i 3}^{2}\right] \frac{M_{3}}{M_{i}} & =-\frac{2 M_{3} m_{\phi}}{\left\langle H_{u}^{0}\right\rangle^{4}} \operatorname{Im}\left[\sum_{j=2}^{3} R_{3 j}^{2} m_{j}^{2}\right] .
\end{aligned}
$$

It is seen that $\left(y_{\nu} y_{\nu}^{\dagger}\right)_{11}$ and $\left(y_{\nu} y_{\nu}^{\dagger}\right)_{22}$ are constrained by the neutrino oscillation data, meanwhile $\left(y_{\nu} y_{\nu}^{\dagger}\right)_{33}$ is basically a free parameter since $R_{3 j}$ is not constrained. This is consistent with the fact that $M_{\nu}$ is independent of $y_{\nu 3 i}$.

The discussion is quite similar in the IH case. The definition of $R$ is the same form as in eq. (4.15), but satisfies $R^{T} R=\operatorname{diag}(1,1,0)$ with $\left(D_{M_{\nu}}^{-1 / 2}\right)^{2}=\operatorname{diag}\left(m_{1}^{-1}, m_{2}^{-1}, 0\right)$. Then, defining $r$ as

$$
r \equiv\left(\begin{array}{ll}
R_{11} & R_{12} \\
R_{21} & R_{22}
\end{array}\right)
$$

we get

$$
\begin{aligned}
r^{T} r & =r r^{T}=\mathbf{1}, \\
R_{41} / R_{31} & =R_{42} / R_{32}=i, \\
R_{l 3} & =0 \quad(l=1-4),
\end{aligned}
$$


and the neutrino Yukawa couplings are given by,

$$
\begin{aligned}
\left(y_{\nu} y_{\nu}^{\dagger}\right)_{i i} & =M_{i} \sum_{j=1}^{2}\left|R_{i j}\right|^{2} m_{j} /\left\langle H_{u}^{0}\right\rangle^{2} \quad(i=1,2) \\
\left(y_{\nu} y_{\nu}^{\dagger}\right)_{33} & =2 m_{\phi} \sum_{j=1}^{2}\left|R_{3 j}\right|^{2} m_{j} /\left\langle H_{u}^{0}\right\rangle^{2} \\
\operatorname{Im}\left[\left(y_{\nu} y_{\nu}^{\dagger}\right)_{21}^{2}\right] \frac{M_{1}}{M_{2}} & =-\frac{M_{1}^{2}}{\left\langle H_{u}^{0}\right\rangle^{4}} \operatorname{Im}\left[\sum_{j=1}^{2} R_{1 j}^{2} m_{j}^{2}\right] \\
\sum_{i=1}^{2} \operatorname{Im}\left[\left(y_{\nu} y_{\nu}^{\dagger}\right)_{i 3}^{2}\right] \frac{M_{3}}{M_{i}} & =-\frac{2 M_{3} m_{\phi}}{\left\langle H_{u}^{0}\right\rangle^{4}} \operatorname{Im}\left[\sum_{j=1}^{2} R_{3 j}^{2} m_{j}^{2}\right]
\end{aligned}
$$

\section{Post inflationary regime}

After the end of inflation, the inflaton oscillates around the global minimum and decays eventually. Due to the decay the universe is reheated and thermal plasma is created. In this section, we estimate the reheating temperature and discuss how the lepton number asymmetry is generated. As in the previous section, we take $\Omega \simeq 1$. After the universe is reheated, gravitinos are produced in various ways. We discuss the gravitino problem at the end of this section.

\section{$5.1 \quad$ Reheating}

The reheating temperature $T_{R}$ due to the inflaton decay is estimated by,

$$
T_{R} \simeq\left(90 / \pi^{2} g_{*}\left(T_{R}\right)\right)^{1 / 4} \sqrt{\Gamma_{\phi} M_{p l}},
$$

where $g_{*}(T)$ is the effective degree of freedom of radiation fields at temperature $T$ and $\Gamma_{\phi}$ is the decay rate of the inflaton. This expression is valid when the neutrino Yukawa couplings that are responsible for the decay is sufficiently small to satisfy $T_{R} \lesssim m_{\phi}[30,31]$, which is the situation we focus on. ${ }^{6}$ The inflaton decays as $\phi \rightarrow L \tilde{H}_{u}, \bar{L} \overline{\tilde{H}}_{u}, \tilde{L} H_{u}, \tilde{L}^{*} H_{u}^{*} \cdot{ }^{7}$ Here flavor indices and $\mathrm{SU}(2)$ doublet components are summed implicitly. Then the decay rates for the modes are given by

$$
\begin{aligned}
& \Gamma_{\phi \rightarrow L \tilde{H}_{u}}=\Gamma_{\phi \rightarrow \bar{L}_{\tilde{H}}}=\frac{\left(y_{\nu} y_{\nu}^{\dagger}\right) 33}{16 \pi} m_{\phi}, \\
& \Gamma_{\phi \rightarrow \tilde{L} H_{u}}=\Gamma_{\phi \rightarrow \tilde{L}^{*} H_{u}^{*}}=\frac{\left(y_{\nu} y_{\nu}^{\dagger}\right)_{33}}{16 \pi} \frac{M_{3}^{2}}{m_{\phi}} .
\end{aligned}
$$

\footnotetext{
${ }^{6}$ Of course, it is possible to consider a higher reheating temperature than the inflaton mass. Such a case is discussed in ref. [24]. We will comment on the impact of such high reheating temperature on leptogenesis in the next subsection.

${ }^{7}$ In general, the inflaton decays to gravitino pair or gravitino and right-handed neutrino. We will discuss those processes in section 5.3.
} 
Since $\Gamma_{\phi \rightarrow \tilde{L} H_{u}}\left(=\Gamma_{\phi \rightarrow \tilde{L}^{*} H_{u}^{*}}\right)$ is suppressed by $\left(M_{3} / m_{\phi}\right)^{2}$, the total decay rate is given by

$$
\Gamma_{\phi} \simeq \Gamma_{\phi \rightarrow L \tilde{H}_{u}}+\Gamma_{\phi \rightarrow \bar{L}_{\tilde{H}}}=\frac{\left(y_{\nu} y_{\nu}^{\dagger}\right)_{33}}{8 \pi} m_{\phi}
$$

Then the reheating temperature is estimated as

$$
T_{R} \simeq 1.4 \times 10^{10} \mathrm{GeV}\left(\frac{m_{\phi}}{10^{13} \mathrm{GeV}}\right)^{1 / 2}\left(\frac{\left(y_{\nu} y_{\nu}^{\dagger}\right)_{33}}{10^{-9}}\right)^{1 / 2}\left(\frac{g_{*}\left(T_{R}\right)}{228.75}\right)^{-1 / 4} .
$$

Recall that $\left(y_{\nu} y_{\nu}^{\dagger}\right)_{33}$ is not constrained by the neutrino observations. As a consequence, it is possible to consider a wide range of values for the reheating temperature, which is suitable for leptogenesis.

To end this subsection, we derive the number of $e$-folds before the end of inflation. In this model, the inflaton oscillates after the end of inflation and eventually decays to reheat the universe. Therefore, it is given by

$$
\begin{aligned}
N_{e} \simeq & 55+\log \left(\frac{L}{\mathrm{Gpc}}\right)+\frac{1}{3} \log \left(\frac{T_{R}}{10^{10} \mathrm{GeV}}\right) \\
& +\log \left(\frac{H_{e}}{10^{13} \mathrm{GeV}}\right)-\frac{2}{3} \log \left(\frac{H_{\text {end }}}{10^{13} \mathrm{GeV}}\right),
\end{aligned}
$$

where $L$ is the present cosmological scale, and $H_{e}$ and $H_{\text {end }}$ are the Hubble scale corresponding to $N_{e}$ and at the end of inflation, respectively. It is now clear that the parameters given in eq. (2.11) is consistent with $N_{e}=55-60$.

\section{$5.2 \quad$ Leptogenesis}

Now we discuss the lepton number asymmetry. The lepton number is generated via leptogenesis [23] (see, for example, refs. [32, 33] for review). In the following numerical study, we discuss following representative cases:

$$
\begin{aligned}
& \text { (I). } M_{1}, M_{2}<m_{\phi}, \\
& \text { (II). } M_{1}, M_{2}>m_{\phi} .
\end{aligned}
$$

In both cases, $M_{3}$ should satisfy eq. (3.23).

Let us consider case (I) first. For simplicity, we consider the Majorana masses are further hierarchical, i.e., $M_{1} \ll M_{2}{ }^{8}$ Similar situation has been studied intensively in the literature [34, 35]. Even though the lepton number is generated by the inflaton decay, it is possibly washed out when the reheating temperature is comparable or higher than $M_{1} \cdot{ }^{9}$

\footnotetext{
${ }^{8} M_{3}$ is irrelevant for leptogenesis if eq. (3.23) is satisfied.

${ }^{9}$ It would be possible that $\tilde{N}_{i}(i=1,2)$ have initial amplitude of the Hubble parameter during inflation $H_{\text {inf }} \sim g \xi / \sqrt{6} M_{p l}$. Then $\tilde{N}_{i}$ start to oscillate when $H \sim M_{i}$, and eventually decays. However, the effect of coherent oscillation of $\tilde{N}_{i}$ is negligible since the energy density ratio of $\tilde{N}_{i}$ to radiation at the decay is estimated as less than $\xi^{2} / 18 M_{p l}^{4} \sim 10^{-9}$.
} 
To see this more explicitly, it is convenient to introduce the effective neutrino mass [36] and equilibrium neutrino mass [37],

$$
\begin{aligned}
& \tilde{m}_{1}=\frac{\left(m_{\nu} m_{\nu}^{\dagger}\right)_{11}}{M_{1}}, \\
& m_{*}=\frac{4 \pi^{2} \sqrt{g_{*}\left(M_{1}\right)}\left\langle H_{u}^{0}\right\rangle^{2}}{3 \sqrt{10} M_{p l}} \simeq 3.9 \times 10^{-4} \mathrm{eV}\left(\frac{\left\langle H_{u}^{0}\right\rangle}{v / 2}\right)^{2},
\end{aligned}
$$

where $v \simeq 246.7 \mathrm{GeV}$. If $\tilde{m}_{1} / m_{*}$ is larger than unity, then it is the strong washout regime and the lepton number generated at the reheating is washed out. $\tilde{m}_{1}$ is estimated by using eqs. (4.21) and (4.29),

$$
\tilde{m}_{1}=\left\{\begin{array}{l}
\sum_{j=2}^{3}\left|R_{1 j}\right|^{2} m_{j}(\mathrm{NH}) \\
\sum_{j=1}^{2}\left|R_{1 j}\right|^{2} m_{j}(\mathrm{IH})
\end{array} .\right.
$$

Using the neutrino mass data and eqs. (4.17) and (4.26), it is straightforward to find that $\tilde{m}_{1}$ has a lower bound,

$$
\tilde{m}_{1} \geq\left\{\begin{array}{l}
m_{2} \simeq 8.6 \times 10^{-3} \mathrm{eV}(\mathrm{NH}) \\
m_{1} \simeq 4.9 \times 10^{-2} \mathrm{eV}(\mathrm{IH})
\end{array} .\right.
$$

Therefore, it is the strong washout regime in either case.

Although the primordial lepton number is washed out, the lepton number is regenerated by the decay of the lightest right-handed (s)neutrino, i.e., $N_{1}$ and $\tilde{N}_{1}$ in the present case. Then the lepton number, strictly speaking lepton number minus baryon number, is converted to baryon number via the sphaleron effect. This scenario works if $T_{R} \gtrsim M_{1}[34,35]$, which is always possible as confirmed in the previous subsection. Then the resultant baryon number becomes independent of $T_{R}$. In our study, we adopt the analytic expressions in ref. [35] for the calculation of the baryon number. Note that although the results there are given in non-supersymmetric model, the results in supersymmetric model do not change much both quantitatively and qualitatively [33, 38, 39]. In our study we adopt the discussion given in ref. [33]. Then the baryon number is determined by

$$
\eta_{B} \equiv \frac{n_{B}}{n_{\gamma}}=\frac{3 \sqrt{2}}{4} \frac{a_{\mathrm{sph}}}{f} \epsilon_{1} \kappa_{f} \simeq 2.7 \times 10^{-10}\left(\frac{\epsilon_{1}}{10^{-6}}\right)\left(\frac{\kappa_{f}}{2 \times 10^{-2}}\right),
$$

where $n_{B}$ and $n_{\gamma}$ are number densities of baryon and photon at present, respectively, $a_{\mathrm{sph}}=28 / 79, f=2387 / 86$, and a factor of $\sqrt{2}$ counts the supersymmetric effect. The efficiency factor $\kappa_{f}$ is given by [35]

$$
\kappa_{f}=(2 \pm 1) \times 10^{-2}\left(\frac{0.01 \mathrm{eV}}{\tilde{m}_{1}}\right)^{1.1 \pm 0.1} .
$$

Finally, referring ref. [40], the asymmetric parameter $\epsilon_{1}$ in our model is given by

$$
\epsilon_{1}=-\frac{3}{16 \pi} \frac{\operatorname{Im}\left[\left(y_{\nu} y_{\nu}^{\dagger}\right)_{21}^{2}\right]}{\left(y_{\nu} y_{\nu}^{\dagger}\right)_{11}} \frac{M_{1}}{M_{2}}=\frac{3}{16 \pi} \frac{M_{1}}{\left\langle H_{u}^{0}\right\rangle^{2}} m_{\mathrm{eff}}
$$




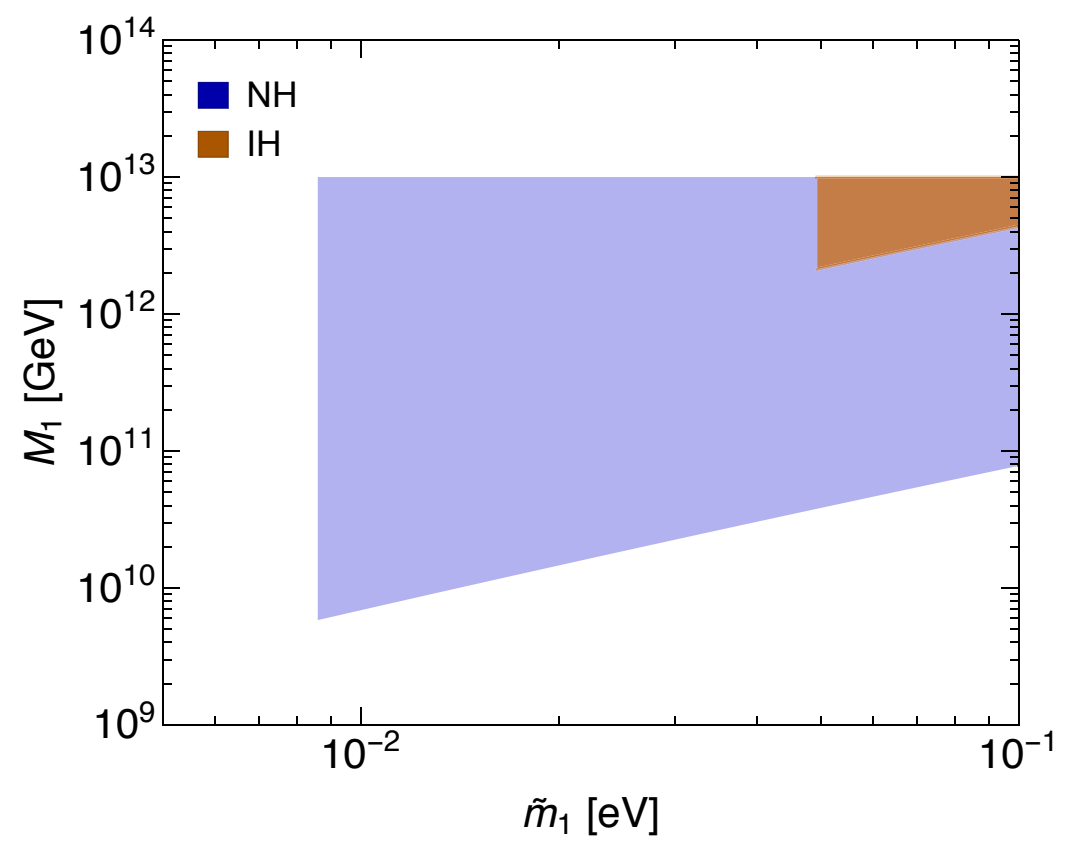

Figure 1. Allowed region for $M_{1}$ as function of effective neutrino mass $\tilde{m}_{1}$ defined in eq. (5.9) for the normal hierarchy $(\mathrm{NH})$ and inverted hierarchy $(\mathrm{IH})$ cases. Lower bound on $M_{1}$ is obtained from $\eta_{B}^{\max } \geq \eta_{B}^{\text {obs }}$ while upper bound is (5.7). Lower bound on $\tilde{m}_{1}$ is given by eq. (5.12).

where we have used eqs. (4.21), (4.23), (4.29), and (4.31) to obtain

$$
m_{\mathrm{eff}}=\left\{\begin{array}{l}
\frac{\operatorname{Im} \sum_{j=2}^{3} R_{1 j}^{2} m_{j}^{2}}{\sum_{j=2}^{3}\left|R_{1 j}\right|^{2} m_{j}}(\mathrm{NH}) \\
\frac{\operatorname{Im} \sum_{j=1}^{2} R_{1 j}^{2} m_{j}^{2}}{\sum_{j=1}^{2}\left|R_{1 j}\right|^{2} m_{j}}(\mathrm{IH})
\end{array} .\right.
$$

It turns out that the maximum value of $m_{\mathrm{eff}}$, denoted as $m_{\mathrm{eff}}^{\max }$, is

$$
m_{\mathrm{eff}}^{\max }=\left\{\begin{array}{l}
m_{3}-m_{2} \simeq 4.2 \times 10^{-2} \mathrm{eV}(\mathrm{NH}) \\
m_{2}-m_{1} \simeq 7.4 \times 10^{-4} \mathrm{eV}(\mathrm{IH})
\end{array} .\right.
$$

Therefore, parametrizing $m_{\mathrm{eff}}$ as $m_{\mathrm{eff}}=m_{\mathrm{eff}}^{\max } \sin \delta$, the asymmetric parameter is given by

$$
\epsilon_{1} \simeq\left\{\begin{array}{l}
8.2 \times 10^{-7}(\mathrm{NH}) \\
1.5 \times 10^{-8}(\mathrm{IH})
\end{array}\right\} \times\left(\frac{M_{1}}{10^{10} \mathrm{GeV}}\right)\left(\frac{\left\langle H_{u}^{0}\right\rangle}{v / 2}\right)^{-2}\left(\frac{\sin \delta}{0.5}\right) .
$$

Using eqs. (5.13), (5.14) and (5.18), the lower limit on $M_{1}$ to explain the present baryon number is determined from $\eta_{B}^{\max } \geq \eta_{B}^{\text {obs }}$ where $\sin \delta=1$ and $\eta_{B}^{\text {obs }}$ is given by [41]

$$
\eta_{B}^{\mathrm{obs}}=(6.12 \pm 0.03) \times 10^{-10} .
$$

In figure 1, allowed regions are depicted for the NH and IH cases. Here we consider socalled high-scale SUSY and take $\left\langle H_{u}^{0}\right\rangle=v / 2$ to get $125 \mathrm{GeV}$ Higgs mass [42, 43]. In the plot upper bound on $M_{1}$ is given by (5.7), i.e., $M_{1}<m_{\phi}=10^{13} \mathrm{GeV}$, and the lower bound on 
$\tilde{m}_{1}$ is from eq. (5.12). ${ }^{10}$ The theoretical uncertainties in eq. (5.14) are taken into account. It is found that the present baryon number can be explained in a wide range of parameter space for the NH case. For the IH case, on the other hand, it seems that the parameter space for a successful leptogenesis is relatively limited. The lowest value required for $M_{1}$ turns out to be

$$
M_{1} \gtrsim\left\{\begin{array}{l}
5.8 \times 10^{9} \mathrm{GeV}(\mathrm{NH}) \\
2.1 \times 10^{12} \mathrm{GeV}(\mathrm{IH})
\end{array} .\right.
$$

The lower limit is near the upper bound in the IH case. Here recall that the upper bound on $M_{1}$ is just a theoretical one. When $M_{1} \sim m_{\phi}, T_{R}$ should be comparable to $m_{\phi}$, which is possible as discussed in refs. [24, 30, 31]. In such a case, sneutrino inflation and leptogenesis can be another source for lepton asymmetry, which will be discussed below in detail. Therefore, the upper bound merely indicates the parameter space for simple thermal leptogenesis to work.

Let us move on to case (II). Since they are much heavier than the inflaton, $N_{1,2}$ and $\tilde{N}_{1,2}$ are never thermalized after the reheating. For $N_{3}$ and $\tilde{N}_{3}$, on the other hand, it depends on the effective neutrino mass that is defined by

$$
\tilde{m}_{3}=\frac{\left(m_{\nu} m_{\nu}^{\dagger}\right)_{33}}{m_{\phi}} \simeq 1.5 \times 10^{-9} \mathrm{eV}\left(\frac{\left(y_{\nu} y_{\nu}^{\dagger}\right)_{33}}{10^{-9}}\right)\left(\frac{10^{13} \mathrm{GeV}}{m_{\phi}}\right)\left(\frac{\left\langle H_{u}^{0}\right\rangle}{v / 2}\right)^{2} .
$$

Then, from eq. (5.5), one obtains

$$
\frac{T_{R}}{m_{\phi}} \simeq 0.71 \times\left(\frac{\tilde{m}_{3}}{m_{*}}\right)^{1 / 2} .
$$

Here $m_{*}$ is defined similarly to eq. (5.10) but replacing $g_{*}\left(M_{1}\right)$ by $g_{*}\left(m_{\phi}\right)$, and we have taken $g_{*}\left(T_{R}\right) \simeq g_{*}\left(m_{\phi}\right)$. As explained in section 5.1, the expression eq. (5.5) is valid for $T_{R} / m_{\phi} \lesssim 1$ that is satisfied for $\tilde{m}_{3}<m_{*}$. Such a case corresponds to the weak washout regime. In that regime, the $N_{3}$ and $\tilde{N}_{3}$ are not thermalized, and the lepton number produced by the inflaton decay can be the source of the present baryon number. This situation is similar to sneutrino inflation and leptogenesis [24, 27, 44-52]. (See also refs. [53-55] for leptogenesis via Afflec-Dine mechanism [56].)

Let us suppose $\tilde{m}_{3} \ll m_{*}$, i.e., $T_{R} / m_{\phi} \ll 1$. Then baryon number is given by

$$
\eta_{B}=\frac{3}{4} \frac{T_{R}}{m_{\phi}} a_{\mathrm{sph}}^{\mathrm{MSSM}} d \epsilon_{\phi},
$$

where $a_{\mathrm{sph}}^{\mathrm{MSSM}}=8 / 23$ and $d=\left(s / n_{\gamma}\right)_{0}=43 \pi^{4} / 495 \zeta(3)$ is the present value of entropy density to and photon density ratio. $\epsilon_{\phi}$ is obtained by an explicit calculation as

$$
\epsilon_{\phi}=-\frac{3}{4 \pi} \sum_{i=1}^{2} \frac{\operatorname{Im}\left[\left(y_{\nu} y_{\nu}^{\dagger}\right)_{i 3}^{2}\right]}{\left(y_{\nu} y_{\nu}^{\dagger}\right)_{33}} \frac{M_{3}}{M_{i}}=\frac{3}{4 \pi} \frac{M_{3}}{\left\langle H_{u}^{0}\right\rangle^{2}} m_{\mathrm{eff}}^{\prime},
$$

\footnotetext{
${ }^{10}$ Strictly speaking, the equality should be excluded since baryon number is zero.
} 
where

$$
m_{\mathrm{eff}}^{\prime}=\left\{\begin{array}{l}
\frac{\operatorname{Im} \sum_{j=2}^{3} R_{3 j}^{2} m_{j}^{2}}{\sum_{j=2}^{3}\left|R_{3 j}\right|^{2} m_{j}}(\mathrm{NH}) \\
\frac{\operatorname{Im} \sum_{j=1}^{2} R_{3 j}^{2} m_{j}^{2}}{\sum_{j=1}^{2}\left|R_{3 j}\right|^{2} m_{j}}(\mathrm{IH})
\end{array} .\right.
$$

In the second step, we have used eqs. (4.22), (4.24), (4.30), and (4.32). It should be noted that $\epsilon_{\phi}$ is independent of the inflaton mass, but it depends on $M_{3}$. Even though $R_{3 j}$ are not constrained, it has been found that $m_{\text {eff }}^{\prime}$ is bounded from above. The maximum value turns out to be

$$
m_{\mathrm{eff}}^{\prime \max }=\left\{\begin{array}{l}
m_{3} \simeq 5.0 \times 10^{-2} \mathrm{eV}(\mathrm{NH}) \\
m_{2} \simeq 5.0 \times 10^{-2} \mathrm{eV}(\mathrm{IH})
\end{array} .\right.
$$

Therefore, parametrizing the effective neutrino mass as $m_{\mathrm{eff}}^{\prime}=m_{\mathrm{eff}}^{\prime \max } \sin \delta^{\prime}$ the asymmetric parameter is given by

$$
\epsilon_{\phi} \simeq 3.9 \times 10^{-9} \times\left(\frac{M_{3}}{10^{7} \mathrm{GeV}}\right)\left(\frac{\left\langle H_{u}^{0}\right\rangle}{v / 2}\right)^{-2}\left(\frac{\sin \delta^{\prime}}{0.5}\right),
$$

for both the $\mathrm{NH}$ and $\mathrm{IH}$ cases. Using the equations, we get

$$
\eta_{B}^{\max } \simeq 1.6 \times 10^{-10}\left(\frac{M_{3}}{10^{7} \mathrm{GeV}}\right)\left(\frac{\tilde{m}_{3}}{10^{-7} \mathrm{eV}}\right)^{1 / 2}\left(\frac{\left\langle H_{u}^{0}\right\rangle}{v / 2}\right)^{-3} .
$$

Since $\eta_{B}$ is independent of $m_{\phi}$, the requirement $\eta_{B}^{\max } \geq \eta_{B}^{\text {obs }}$ gives a lower bound on $M_{3}$, which is plotted in figure 2. Here $\left\langle H_{u}^{0}\right\rangle=v / 2$ is taken as in figure 1. Upper bound $M_{3}<2 \times 10^{11} \mathrm{GeV}$ is from eq. (3.23). Another upper bound on $M_{3}$ from the stability of the inflationary trajectory, eq. (3.28), is also shown. We quit plotting for region $\tilde{m}_{3}>10^{-5} \mathrm{eV}$ because $\tilde{m}_{3} \ll m_{*}$ is no longer valid. In the plot, contours of $T_{R}$ are depicted. It is found that the leptogenesis is successful in a wide parameter space for both the NH and IH cases. Lower bound on $M_{3}$ behaves similarly to region $\mathrm{C}$ in figure 1 of ref. [48] by reading $M_{1}$ and $\tilde{m}_{1}$ as $M_{3}$ and $\tilde{m}_{3}$, respectively, i.e., the lower bound is proportional to $1 / \sqrt{\tilde{m}_{3}}$. Quantitatively, the lower bound in our model is relaxed by roughly a factor of 4 compared to the result in the reference. This can be understood as follows; first, the decay rate of the inflaton in our model is different from one in the literature (see eq. (5.4)), leading to a $1 / \sqrt{2}$ suppression in $T_{R} / m_{\phi}$; as another consequence, the expression (5.24) (with $m_{\mathrm{eff}}^{\prime}=m_{\mathrm{eff}}^{\prime \max }$ ) is enhanced by a factor of two compared to $\left|\epsilon_{1}^{\max }\right|$ in the reference; finally $\tan \beta=\infty$ is taken in the work meanwhile $\tan \beta=1$ in our model. Thus, in total, a factor of 4 enhancement is obtained in $\eta_{B}$.

In the case where $\tilde{m}_{3}$ gets much larger than $m_{*}$, the situation reduces to case (I). Namely, the reheating temperature is so high that both $\tilde{N}_{3}$ and $N_{3}$ are thermalized and thermal leptogenesis takes place. Resultant allowed region is the same as the NH case of figure 1 , by replacing $M_{1}$ and $\tilde{m}_{1}$ by $M_{3}$ and $\tilde{m}_{3}$, respectively, but there is no lower bound on $\tilde{m}_{3}$ meanwhile there is the upper bound on $M_{3}$. In the intermediate case, $\tilde{m}_{3} \sim m_{*}$, on the other hand, the Boltzmann equations should be solved numerically to get the lepton 


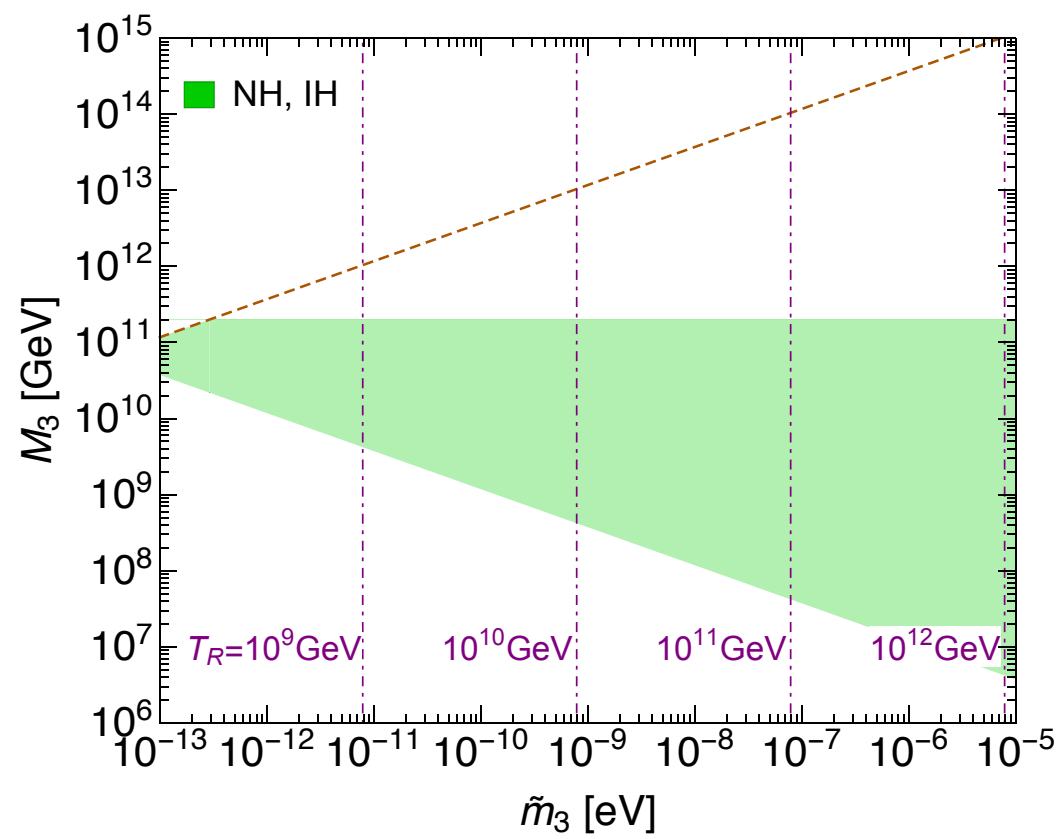

Figure 2. Allowed region for $M_{3}$ as function of $\tilde{m}_{3}$ defined in eq. (5.21). Results are the same for the $\mathrm{NH}$ and $\mathrm{IH}$ cases. Lower bound on $M_{3}$ comes from $\eta_{B}^{\max } \geq \eta_{B}^{\text {obs }}$ meanwhile upper one is given by eqs. (3.23) and (3.28). The former is not to affect the inflationary trajectory and the latter (dashed orange) is from the stability of the scalar potential. Vertical lines (dot-dashed purple) are contours of $T_{R}$.

number, which is already done in ref. [48]. The result corresponds to region B in figure 1 of the reference. Strictly speaking, the effective dissipation rate should be used instead of the decay rate of the inflaton [31] in the Boltzmann equations. As shown in the reference, the reheating process is so efficient when the dissipation rate is taken into account that the reheating temperature can exceed the mass of the inflaton mass and consequently $N_{3}$ and $\tilde{N}_{3}$ are easily thermalized. Once they are thermalized, the thermal leptogenesis takes place, where the resultant baryon number becomes independent of the reheating temperature. Eventually the situation reduces to the case (I). Such qualitative behavior can be confirmed by numerical study, which is left for the future work.

Crucial difference from sneutrino leptogenesis [44-48] is that although $M_{3}$ and $\tilde{m}_{3}$, i.e., $\left(y_{\nu} y_{\nu}^{\dagger}\right)_{33}$, are important parameters to determine baryon number, they are sequestered from other physical quantities, such as the heavy right-handed (s)neutrino masses or the light neutrino mass matrix. Therefore, there is no consequence in other low energy experiments. This is a feature of case (II).

\subsection{Gravitino problem}

In the framework of supergravity, a fair amount of gravitino $\psi_{\mu}$ can be produced in various ways in the thermal history of the universe. Since the interactions of gravitino with the MSSM particles are Planck-suppressed, gravitino is long-lived and its decay can spoil the successful big-bang nucleosynthesis if it is unstable. Although this problem can be avoided 
when gravitino is enough heavy to have the lifetime much shorter than $1 \mathrm{sec}$, gravitino decay produces the lightest superparticle (LSP). Then the LSP produced by the decay may overclose the universe if the R-parity is conserved.

There are three types of production mechanism of gravitino in the model we consider; (i) the inflaton decay; (ii) thermal scattering from the thermal bath [57-60]; (iii) decay of superparticles in the thermal bath $[61,62]$.

In general, process (i) includes gravitino pair production. The decay width of the mode, however, depends on the inflaton VEV [63-68]. In our case, therefore, this process can be ignored since the inflaton does not have a VEV. On the other hand, the inflaton can decay to gravitino and right-handed neutrino. The decay width is given by

$$
\Gamma_{\phi \rightarrow \psi_{\mu} N_{3}}=\frac{\beta_{f}^{3} m_{\phi}^{5}}{48 \pi M_{p l}^{2} m_{3 / 2}^{2}}\left[1-\frac{\left(m_{f}+m_{3 / 2}\right)^{2}}{m_{\phi}^{2}}\right],
$$

where $\beta_{f}^{2}=1-2\left(m_{f}^{2}+m_{3 / 2}^{2}\right) / m_{\phi}^{2}+\left(m_{f}^{2}-m_{3 / 2}^{2}\right)^{2} / m_{\phi}^{4}$. Here $m_{f}$ and $m_{3 / 2}$ are masses of $N_{3}$ and $\psi_{\mu}$, respectively. The mass difference between $\phi$ and $N_{3}$ is expected to be given by the soft SUSY breaking mass scale for scalar superpartners, $\left|m_{\phi}-m_{f}\right| \sim \tilde{m}$. Let us assume this decay happens by taking $m_{\phi}=m_{f}+\tilde{m}$ where $\tilde{m}=k m_{3 / 2}(k>1)$. In the limit $k m_{3 / 2} / m_{\phi} \ll 1$, we obtain

$$
\Gamma_{\phi \rightarrow \psi_{\mu} N_{3}} \simeq \frac{C m_{\phi} m_{3 / 2}^{2}}{3 \pi M_{p l}^{2}}
$$

where $C=(k-1)\left(k^{2}-1\right)^{3 / 2}$. Then the branching fraction of this mode is

$$
\mathrm{Br}_{\phi \rightarrow \psi_{\mu} N_{3}}=\frac{\Gamma_{\phi \rightarrow \psi_{\mu} N_{3}}}{\Gamma_{\phi}} \simeq 4.5 \times 10^{-16} C\left(\frac{m_{3 / 2}}{10^{5} \mathrm{GeV}}\right)^{2}\left(\frac{10^{-11}}{\left(y_{\nu} y_{\nu}^{\dagger}\right)_{33}}\right) .
$$

Therefore, it is sure that the inflaton decay reheats the universe in wide range of gravitino mass region. On the other hand, the resultant gravitino abundance produced by the decay is estimated as

$$
\Omega_{3 / 2}^{\inf } h^{2} \sim 1.3 \times 10^{-6} C\left(\frac{m_{3 / 2}}{10^{5} \mathrm{GeV}}\right)^{3}\left(\frac{10^{13} \mathrm{GeV}}{m_{\phi}}\right)^{1 / 2}\left(\frac{10^{-11}}{\left(y_{\nu} y_{\nu}^{\dagger}\right)_{33}}\right)^{1 / 2},
$$

where $h$ is the scale factor of Hubble expansion rate.

Gravitino abundance via process (ii) is most effective at high temperature, thus it is proportional to $T_{R}$, meanwhile in process (iii) gravitino is dominantly produced when the temperature is around the mass of decaying particle. Adopting the expression given in ref. [69], the abundances via processes (ii) and (iii) are given by

$$
\begin{aligned}
& \Omega_{3 / 2}^{\mathrm{TH}} h^{2} \sim 4\left(\frac{T_{R}}{10^{9} \mathrm{GeV}}\right)\left(\frac{m_{3 / 2}}{10^{5} \mathrm{GeV}}\right), \\
& \Omega_{3 / 2}^{\mathrm{FI}} h^{2} \sim 5 \times 10^{-4} k^{3}\left(\frac{m_{3 / 2}}{10^{5} \mathrm{GeV}}\right)^{2} .
\end{aligned}
$$



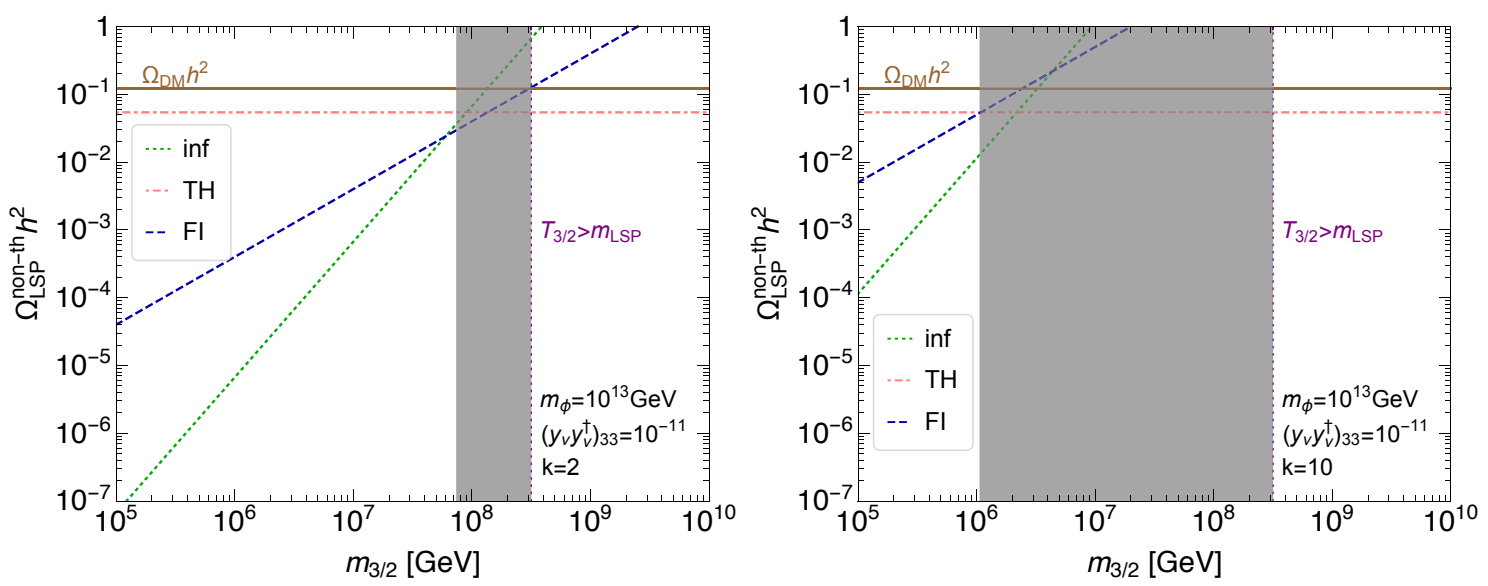

Figure 3. Relic density of the LSP as function of gravitino mass. "inf" (dotted green), "TH" (dot-dashed red), and "FI" (dashed blue) are the contributions from process (i), (ii), and (iii), respectively. $\Omega_{\mathrm{DM}} h^{2} \simeq 0.12$ (solid brown) is indicated as a reference. Right region from vertical line (dotted violet) indicates $T_{3 / 2}>m_{\mathrm{LSP}}$, and shaded region is excluded. $m_{\mathrm{LSP}}=1 \mathrm{TeV}$, $m_{\phi}=10^{13} \mathrm{GeV},\left(y_{\nu} y_{\nu}^{\dagger}\right)_{33}=10^{-11}$, and $k=\tilde{m} / m_{3 / 2}=2$ (left), 10 (right) are taken.

Here the contribution of the longitudinal mode of gravitino is suppressed in $\Omega_{3 / 2}^{\mathrm{TH}}$ by considering gluino is lighter than gravitino. In $\Omega_{3 / 2}^{\mathrm{FI}}$, we have assumed that all scalar leptons and quarks in the MSSM (whose mass scale is $\tilde{m}$ ) are heavier than gravitino and that they are thermalized. And as in the discussion of the inflaton decay, $\tilde{m}=k m_{3 / 2}$ has been taken. ${ }^{11}$ Then the relic abundance of the LSP due to gravitino decay is estimated as

$$
\Omega_{\mathrm{LSP}}^{\text {non-th }} h^{2}=\frac{m_{\mathrm{LSP}}}{m_{3 / 2}} \Omega_{3 / 2} h^{2},
$$

where $m_{\mathrm{LSP}}$ is the LSP mass and $\Omega_{3 / 2}=\Omega_{3 / 2}^{\mathrm{inf}}+\Omega_{3 / 2}^{\mathrm{TH}}+\Omega_{3 / 2}^{\mathrm{FI}}$ is the total gravitino abundance. $\Omega_{\mathrm{LSP}}^{\text {non-th }} h^{2}$ should not exceed the observed dark matter abundance $\Omega_{\mathrm{DM}} h^{2} \simeq 0.12$ [41], which gives a constraint on gravitino mass.

Figure 3 shows the resultant $\Omega_{\mathrm{LSP}}^{\text {non-th }}$ from the each contribution. Here $m_{\mathrm{LSP}}=1 \mathrm{TeV}$ is taken by considering $1 \mathrm{TeV}$ Higgsino or Wino dark matter with a mass of $2.7-3 \mathrm{TeV}$ [70]. $m_{\phi}=10^{13} \mathrm{GeV},\left(y_{\nu} y_{\nu}^{\dagger}\right)_{33}=10^{-11}$, and $k=2$ (left), 10 (right) are taken to determine the contributions from processes (i) and (iii). It is seen that in lower gravitino mass region, the dominant contribution to $\Omega_{\mathrm{LSP}}^{\text {non-th }}$ is from process (ii). In order for the contribution not to exceed the dark matter abundance, $T_{R} \lesssim 10^{9} \mathrm{GeV}$ is required, which is well-known result. This gives a stringent constraint on the parameter space for successful leptogenesis shown in figures 1 and 2 if gravitino is not heavy enough. Namely, $\tilde{m}_{1} \sim 10^{-2} \mathrm{eV}$ is the allowed region in case (I) meanwhile $10^{-13} \mathrm{eV} \lesssim \tilde{m}_{3} \lesssim 10^{-11} \mathrm{eV}$ is allowed in case (II). On the other hand, the contributions from processes (i) and (iii) depend on the parameters, especially $k=\tilde{m} / m_{3 / 2}$ (and $m_{3 / 2}$ ). In order for $\Omega_{\mathrm{LSP}}^{\text {non-th }}$ not to exceed the dark matter abundance, the upper bound on gravitino mass is obtained depending on the mass spectrum of squarks and sleptons, e.g., $m_{3 / 2} \lesssim 10^{8}\left(10^{6}\right) \mathrm{GeV}$ for $\tilde{m} \sim m_{3 / 2}\left(10 m_{3 / 2}\right)$. Such gravitino mass is

\footnotetext{
${ }^{11}$ We have checked that the contribution from $\tilde{N}_{1}$ is negligible even if $\tilde{N}_{1}$ is thermalized, i.e., in case (I).
} 
preferred in minimal or mini split supersymmetry [71, 72], pure gravity mediation [73, 74], and spread supersymmetry $[69,75]$.

On the other hand, there is also an allowed region in higher gravitino mass region. This is because gravitino decays before thermal freeze-out of the LSP in that region. The allowed region can be estimated by imposing gravitino decay temperature $T_{3 / 2}$ larger than the LSP mass. The gravitino decay temperature is defined by

$$
T_{3 / 2} \simeq\left(90 / \pi^{2} g_{*}\left(T_{3 / 2}\right)\right)^{1 / 4} \sqrt{\Gamma_{3 / 2} M_{p l}}
$$

where $\Gamma_{3 / 2}$ is the decay rate of gravitino. Then $m_{3 / 2} \gtrsim 3 \times 10^{8} \mathrm{GeV}$ is obtained from $T_{3 / 2}>m_{\mathrm{LSP}}$ for $m_{\mathrm{LSP}}=1 \mathrm{TeV}$ (see e.g., ref. [76]). Such high gravitino mass can be considered high-scale SUSY [77], intermediate scale supersymmetry [78], and unified inflation model [16]. However, gravitino cannot be too heavy because $\mathrm{Br}_{\phi \rightarrow \psi_{\mu} N_{3}}$ should be less than unity for the reheating. Taking $\mathrm{Br}_{\phi \rightarrow \psi_{\mu} N_{3}} \lesssim 0.1$, for example, upper bound on gravitino mass is obtained as $m_{3 / 2} \lesssim 5 \times 10^{11} \mathrm{GeV}\left(2 \times 10^{10} \mathrm{GeV}\right)$ for $k=2(10)$.

Another option is the R-parity violation. Under the R-parity violation, the LSP decays to the standard-model particles. Then, the LSP does not contribute to the matter abundance of the universe so that there is no constraint on $m_{3 / 2}$.

\section{Conclusion}

Superconformal subcritical hybrid inflation is one of attractive inflation models that are consistent with the observed cosmological parameters by the Planck satellite. In this paper we have studied the cosmology of an extended version of the model. In the model three right-handed neutrinos are introduced. The superpotential consists of one in the supersymmetric seesaw model and the interaction terms of the right-handed neutrinos with the additional matter fields, one of which plays the role of the waterfall field. In the Kähler potential, on the other hand, it is possible for the sneutrinos to have shift symmetry by introducing explicit superconformal breaking terms of $\mathcal{O}(1)$. Due to the shift symmetry, one of the sneutrinos becomes the inflaton field similarly in superconformal subcritical hybrid inflation. Although the mass terms of the sneutrinos can affect the trajectory of the inflaton, it has turned out that the effect is restrictive and viable inflation is realized. After inflation, the inflaton field decays to Higgses and sleptons or Higgsinos and leptons to reheat the universe.

Light neutrino masses are given by the seesaw mechanism. However, the mass matrix is different from the conventional one. It turns out that one of the neutrinos is massless. Assuming that suppressed couplings of the other right-handed neutrinos to the waterfall field, it has been found that the neutrino Yukawa couplings that couples the inflaton to the MSSM sector are not constrained by the neutrino oscillation data. Consequently, the reheating temperature is a free parameter, which is suitable for leptogenesis.

We have considered two representative cases; (I) the other right-handed (s)neutrinos are lighter than the inflaton; (II) the other right-handed (s)neutrinos are heavier than the inflaton. In case (I), thermal leptogenesis is possible if the reheating temperature is larger 
than $\sim 10^{9} \mathrm{GeV}$. It has been found leptogenesis is successful in a wide range of parameter space in the normal hierarchy case while the parameter space for leptogenesis is relatively limited in the inverted hierarchy case. In case (II), on the other hand, sneutrino leptogenesis takes place if the reheating temperature is larger than $\sim 10^{8} \mathrm{GeV}$. It has turned out that in both the normal and inverted hierarchy cases successful leptogenesis is realized in wide range of parameter space.

\section{Acknowledgments}

We are grateful to Wilfried Buchmüller, Pasquale Di Bari, Oleg Lebedev, and Fuminobu Takahashi for valuable discussions. This work was supported by JSPS KAKENHI Grant Numbers JP17H05402, JP17K14278, JP17H02875 and JP18H05542 (KI).

Open Access. This article is distributed under the terms of the Creative Commons Attribution License (CC-BY 4.0), which permits any use, distribution and reproduction in any medium, provided the original author(s) and source are credited.

\section{References}

[1] M. Kawasaki, M. Yamaguchi and T. Yanagida, Natural chaotic inflation in supergravity, Phys. Rev. Lett. 85 (2000) 3572 [hep-ph/0004243] [INSPIRE].

[2] W. Buchmüller, V. Domcke and K. Schmitz, The Chaotic Regime of D-Term Inflation, JCAP 11 (2014) 006 [arXiv: 1406.6300] [INSPIRE].

[3] W. Buchmüller and K. Ishiwata, Grand Unification and Subcritical Hybrid Inflation, Phys. Rev. D 91 (2015) 081302 [arXiv:1412.3764] [INSPIRE].

[4] W. Buchmüller, V. Domcke and K. Schmitz, Superconformal D-Term Inflation, JCAP 04 (2013) 019 [arXiv: 1210.4105] [INSPIRE].

[5] W. Buchmüller, V. Domcke and K. Kamada, The Starobinsky Model from Superconformal D-Term Inflation, Phys. Lett. B 726 (2013) 467 [arXiv: 1306.3471] [INSPIRE].

[6] A.A. Starobinsky, A New Type of Isotropic Cosmological Models Without Singularity, Phys. Lett. B 91 (1980) 99 [INSPIRE].

[7] M.B. Einhorn and D.R.T. Jones, Inflation with Non-minimal Gravitational Couplings in Supergravity, JHEP 03 (2010) 026 [arXiv:0912.2718] [INSPIRE].

[8] R. Kallosh and A. Linde, New models of chaotic inflation in supergravity, JCAP 11 (2010) 011 [arXiv: 1008.3375] [INSPIRE].

[9] S. Ferrara, R. Kallosh, A. Linde, A. Marrani and A. Van Proeyen, Jordan Frame Supergravity and Inflation in NMSSM, Phys. Rev. D 82 (2010) 045003 [arXiv: 1004.0712] [INSPIRE].

[10] S. Ferrara, R. Kallosh, A. Linde, A. Marrani and A. Van Proeyen, Superconformal Symmetry, NMSSM and Inflation, Phys. Rev. D 83 (2011) 025008 [arXiv:1008.2942] [InSPIRE].

[11] R. Kallosh, A. Linde and D. Roest, Superconformal Inflationary $\alpha$-Attractors, JHEP 11 (2013) 198 [arXiv:1311.0472] [INSPIRE].

[12] R. Kallosh and A. Linde, Superconformal generalizations of the Starobinsky model, JCAP 06 (2013) 028 [arXiv:1306.3214] [INSPIRE]. 
[13] K. Ishiwata, Superconformal Subcritical Hybrid Inflation, Phys. Lett. B 782 (2018) 367 [arXiv: 1803.08274] [INSPIRE].

[14] B.C. Bryant and S. Raby, Pati-Salam version of subcritical hybrid inflation, Phys. Rev. D 93 (2016) 095003 [arXiv:1601.03749] [INSPIRE].

[15] B.C. Bryant, Z. Poh and S. Raby, Reheating and Leptogenesis after Pati-Salam F-term Subcritical Hybrid Inflation, arXiv:1612.04382 [INSPIRE].

[16] V. Domcke and K. Schmitz, Inflation from High-Scale Supersymmetry Breaking, Phys. Rev. D 97 (2018) 115025 [arXiv:1712.08121] [INSPIRE].

[17] P. Minkowski, $\mu \rightarrow$ er at a Rate of One Out of $10^{9}$ Muon Decays?, Phys. Lett. 67B (1977) 421 [INSPIRE].

[18] T. Yanagida, Horizontal gauge symmetry and masses of neutrinos, Conf. Proc. C $\mathbf{7 9 0 2 1 3 1}$ (1979) 95 [INSPIRE].

[19] T. Yanagida, Horizontal Symmetry and Masses of Neutrinos, Prog. Theor. Phys. 64 (1980) 1103 [INSPIRE].

[20] M. Gell-Mann, P. Ramond and R. Slansky, Complex Spinors and Unified Theories, Conf. Proc. C 790927 (1979) 315 [arXiv:1306.4669] [INSPIRE].

[21] P. Ramond, The Family Group in Grand Unified Theories, [hep-ph/9809459] [inSPIRE].

[22] S.L. Glashow, The Future of Elementary Particle Physics, NATO Sci. Ser. B 61 (1980) 687 [INSPIRE].

[23] M. Fukugita and T. Yanagida, Baryogenesis Without Grand Unification, Phys. Lett. B 174 (1986) 45 [INSPIRE].

[24] K. Nakayama, F. Takahashi and T.T. Yanagida, Viable Chaotic Inflation as a Source of Neutrino Masses and Leptogenesis, Phys. Lett. B 757 (2016) 32 [arXiv:1601.00192] [INSPIRE].

[25] NuFIT 4.0 (2018) [www.nu-fit.org].

[26] I. Esteban, M.C. Gonzalez-Garcia, A. Hernandez-Cabezudo, M. Maltoni and T. Schwetz, Global analysis of three-flavour neutrino oscillations: synergies and tensions in the determination of $\theta_{23}, \delta_{C P}$ and the mass ordering, JHEP 01 (2019) 106 [arXiv: 1811.05487] [INSPIRE].

[27] F. Björkeroth, S.F. King, K. Schmitz and T.T. Yanagida, Leptogenesis after Chaotic Sneutrino Inflation and the Supersymmetry Breaking Scale, Nucl. Phys. B 916 (2017) 688 [arXiv: 1608.04911] [INSPIRE].

[28] J.A. Casas and A. Ibarra, Oscillating neutrinos and $\mu \rightarrow e, \gamma$, Nucl. Phys. B 618 (2001) 171 [hep-ph/0103065] [INSPIRE].

[29] S. Davidson and A. Ibarra, A Lower bound on the right-handed neutrino mass from leptogenesis, Phys. Lett. B 535 (2002) 25 [hep-ph/0202239] [INSPIRE].

[30] K. Mukaida and K. Nakayama, Dynamics of oscillating scalar field in thermal environment, JCAP 01 (2013) 017 [arXiv: 1208.3399] [INSPIRE].

[31] K. Mukaida and K. Nakayama, Dissipative Effects on Reheating after Inflation, JCAP 03 (2013) 002 [arXiv:1212.4985] [INSPIRE]. 
[32] W. Buchmüller, R.D. Peccei and T. Yanagida, Leptogenesis as the origin of matter, Ann. Rev. Nucl. Part. Sci. 55 (2005) 311 [hep-ph/0502169] [InSPIRE].

[33] S. Davidson, E. Nardi and Y. Nir, Leptogenesis, Phys. Rept. 466 (2008) 105 [arXiv: 0802.2962] [INSPIRE].

[34] G.F. Giudice, A. Notari, M. Raidal, A. Riotto and A. Strumia, Towards a complete theory of thermal leptogenesis in the SM and MSSM, Nucl. Phys. B 685 (2004) 89 [hep-ph/0310123] [INSPIRE].

[35] W. Buchmüller, P. Di Bari and M. Plümacher, Leptogenesis for pedestrians, Annals Phys. 315 (2005) 305 [hep-ph/0401240] [INSPIRE].

[36] M. Plümacher, Baryogenesis and lepton number violation, Z. Phys. C 74 (1997) 549 [hep-ph/9604229] [INSPIRE].

[37] E.W. Kolb and M.S. Turner, The Early Universe, Addison-Wesley, New York (1990).

[38] M. Plümacher, Baryon asymmetry, neutrino mixing and supersymmetric $\mathrm{SO}(10)$ unification, Nucl. Phys. B 530 (1998) 207 [hep-ph/9704231] [inSPIRE].

[39] B.A. Campbell, S. Davidson and K.A. Olive, Inflation, neutrino baryogenesis and (S)neutrino induced baryogenesis, Nucl. Phys. B 399 (1993) 111 [hep-ph/9302223] [INSPIRE].

[40] L. Covi, E. Roulet and F. Vissani, CP violating decays in leptogenesis scenarios, Phys. Lett. B 384 (1996) 169 [hep-ph/9605319] [INSPIRE].

[41] Planck collaboration, Planck 2018 results. VI. Cosmological parameters, arXiv: 1807.06209 [INSPIRE].

[42] G.F. Giudice and A. Romanino, Split supersymmetry, Nucl. Phys. B 699 (2004) 65 [Erratum ibid. B 706 (2005) 487] [hep-ph/0406088] [INSPIRE].

[43] G.F. Giudice and A. Strumia, Probing High-Scale and Split Supersymmetry with Higgs Mass Measurements, Nucl. Phys. B 858 (2012) 63 [arXiv:1108.6077] [INSPIRE].

[44] H. Murayama, H. Suzuki, T. Yanagida and J. Yokoyama, Chaotic inflation and baryogenesis by right-handed sneutrinos, Phys. Rev. Lett. 70 (1993) 1912 [InSPIRE].

[45] H. Murayama, H. Suzuki, T. Yanagida and J. Yokoyama, Chaotic inflation and baryogenesis in supergravity, Phys. Rev. D 50 (1994) R2356 [hep-ph/9311326] [INSPIRE].

[46] H. Murayama and T. Yanagida, Leptogenesis in supersymmetric standard model with right-handed neutrino, Phys. Lett. B 322 (1994) 349 [hep-ph/9310297] [INSPIRE].

[47] K. Hamaguchi, H. Murayama and T. Yanagida, Leptogenesis from $N$ dominated early universe, Phys. Rev. D 65 (2002) 043512 [hep-ph/0109030] [InSPIRE].

[48] J.R. Ellis, M. Raidal and T. Yanagida, Sneutrino inflation in the light of WMAP: Reheating, leptogenesis and flavor violating lepton decays, Phys. Lett. B 581 (2004) 9 [hep-ph/0303242] [INSPIRE].

[49] S. Antusch, M. Bastero-Gil, S.F. King and Q. Shafi, Sneutrino hybrid inflation in supergravity, Phys. Rev. D 71 (2005) 083519 [hep-ph/0411298] [INSPIRE].

[50] S. Antusch, M. Bastero-Gil, K. Dutta, S.F. King and P.M. Kostka, Chaotic Inflation in Supergravity with Heisenberg Symmetry, Phys. Lett. B 679 (2009) 428 [arXiv:0905.0905] [INSPIRE]. 
[51] K. Kadota and J. Yokoyama, D-term inflation and leptogenesis by right-handed sneutrino, Phys. Rev. D 73 (2006) 043507 [hep-ph/0512221] [INSPIRE].

[52] K. Nakayama, F. Takahashi and T.T. Yanagida, Chaotic Inflation with Right-handed Sneutrinos after Planck, Phys. Lett. B 730 (2014) 24 [arXiv:1311.4253] [INSPIRE].

[53] P. Binetruy and M.K. Gaillard, Noncompact Symmetries and Scalar Masses in Superstring-Inspired Models, Phys. Lett. B 195 (1987) 382 [INSPIRE].

[54] M.K. Gaillard, H. Murayama and K.A. Olive, Preserving flat directions during inflation, Phys. Lett. B 355 (1995) 71 [hep-ph/9504307] [INSPIRE].

[55] J.L. Evans, T. Gherghetta and M. Peloso, Affleck-Dine Sneutrino Inflation, Phys. Rev. D 92 (2015) 021303 [arXiv:1501.06560] [INSPIRE].

[56] I. Affleck and M. Dine, A New Mechanism for Baryogenesis, Nucl. Phys. B 249 (1985) 361 [INSPIRE].

[57] M. Bolz, A. Brandenburg and W. Buchmüller, Thermal production of gravitinos, Nucl. Phys. B 606 (2001) 518 [Erratum ibid. B 790 (2008) 336] [hep-ph/0012052] [INSPIRE].

[58] J. Pradler and F.D. Steffen, Thermal gravitino production and collider tests of leptogenesis, Phys. Rev. D 75 (2007) 023509 [hep-ph/0608344] [INSPIRE].

[59] J. Pradler and F.D. Steffen, Constraints on the Reheating Temperature in Gravitino Dark Matter Scenarios, Phys. Lett. B 648 (2007) 224 [hep-ph/0612291] [INSPIRE].

[60] V.S. Rychkov and A. Strumia, Thermal production of gravitinos, Phys. Rev. D 75 (2007) 075011 [hep-ph/0701104] [INSPIRE].

[61] T. Asaka, K. Ishiwata and T. Moroi, Right-handed sneutrino as cold dark matter, Phys. Rev. D 73 (2006) 051301 [hep-ph/0512118] [INSPIRE].

[62] C. Cheung, G. Elor and L. Hall, Gravitino Freeze-In, Phys. Rev. D 84 (2011) 115021 [arXiv: 1103.4394] [INSPIRE].

[63] M. Kawasaki, F. Takahashi and T.T. Yanagida, Gravitino overproduction in inflaton decay, Phys. Lett. B 638 (2006) 8 [hep-ph/0603265] [INSPIRE].

[64] M. Kawasaki, F. Takahashi and T.T. Yanagida, The Gravitino-overproduction problem in inflationary universe, Phys. Rev. D 74 (2006) 043519 [hep-ph/0605297] [INSPIRE].

[65] T. Asaka, S. Nakamura and M. Yamaguchi, Gravitinos from heavy scalar decay, Phys. Rev. D 74 (2006) 023520 [hep-ph/0604132] [INSPIRE].

[66] M. Endo, K. Hamaguchi and F. Takahashi, Moduli/Inflaton Mixing with Supersymmetry Breaking Field, Phys. Rev. D 74 (2006) 023531 [hep-ph/0605091] [INSPIRE].

[67] M. Endo, F. Takahashi and T.T. Yanagida, Anomaly-induced inflaton decay and gravitino-overproduction problem, Phys. Lett. B 658 (2008) 236 [hep-ph/0701042] [INSPIRE].

[68] M. Endo, F. Takahashi and T.T. Yanagida, Inflaton Decay in Supergravity, Phys. Rev. D 76 (2007) 083509 [arXiv:0706.0986] [INSPIRE].

[69] L.J. Hall, Y. Nomura and S. Shirai, Spread Supersymmetry with Wino LSP: Gluino and Dark Matter Signals, JHEP 01 (2013) 036 [arXiv:1210.2395] [INSPIRE].

[70] J. Hisano, S. Matsumoto, M. Nagai, O. Saito and M. Senami, Non-perturbative effect on thermal relic abundance of dark matter, Phys. Lett. B 646 (2007) 34 [hep-ph/0610249] [INSPIRE]. 
[71] A. Arvanitaki, N. Craig, S. Dimopoulos and G. Villadoro, Mini-Split, JHEP 02 (2013) 126 [arXiv: 1210.0555] [INSPIRE].

[72] N. Arkani-Hamed, A. Gupta, D.E. Kaplan, N. Weiner and T. Zorawski, Simply Unnatural Supersymmetry, arXiv:1212.6971 [INSPIRE].

[73] M. Ibe and T.T. Yanagida, The Lightest Higgs Boson Mass in Pure Gravity Mediation Model, Phys. Lett. B 709 (2012) 374 [arXiv:1112.2462] [INSPIRE].

[74] M. Ibe, S. Matsumoto and T.T. Yanagida, Pure Gravity Mediation with $m_{3 / 2}=10-100 \mathrm{TeV}$, Phys. Rev. D 85 (2012) 095011 [arXiv:1202.2253] [INSPIRE].

[75] L.J. Hall and Y. Nomura, Spread Supersymmetry, JHEP 01 (2012) 082 [arXiv:1111.4519] [INSPIRE].

[76] K. Ishiwata, K.S. Jeong and F. Takahashi, Moduli-induced Baryogenesis, JHEP 02 (2014) 062 [arXiv: 1312.0954] [INSPIRE].

[77] L.J. Hall and Y. Nomura, A Finely-Predicted Higgs Boson Mass from A Finely-Tuned Weak Scale, JHEP 03 (2010) 076 [arXiv:0910.2235] [INSPIRE].

[78] L.J. Hall, Y. Nomura and S. Shirai, Grand Unification, Axion and Inflation in Intermediate Scale Supersymmetry, JHEP 06 (2014) 137 [arXiv:1403.8138] [INSPIRE]. 